\title{
Empirical Bayes functional models for hydrogen deuterium exchange mass spectrometry
}

\author{
Oliver M. Crook *1, Chun-wa Chung ${ }^{2}$, and Charlotte M. Deane ${ }^{1}$ \\ ${ }^{1}$ Department of Statistics, University of Oxford, Oxford, UK \\ ${ }^{2}$ Structural and Biophysical Sciences, GlaxoSmithKline RछD, Stevenage, UK
}

October 18, 2021

\begin{abstract}
A protein's structure is state-specific and a key determinant of its function. Proteins can undergo subtle structural changes when binding to another protein, small molecule or as a result of environmental perturbations. Hydrogen deuterium exchange mass spectrometry (HDX-MS) is a technique to explore differential protein structure by examining the rate of deuterium incorporation for specific peptides. This rate will be altered upon structural perturbation and detecting significant changes to this rate requires a statistical test. To determine rates of incorporation, HDX-MS measurements are frequently made over a time course. However, current statistical testing procedures ignore the correlations in the temporal dimension of the data. Using tools from functional data analysis, we develop a testing procedure that explicitly incorporates a physical model of hydrogen deuterium exchange. To further improve statistical power, we develop an empirical Bayes version of our method, allowing us to borrow information across peptides and stabilise variance estimates for low sample sizes. Our approach has increased power, reduces false positives and improves interpretation over linear model-based approaches. Due to the improved flexibility of our method, we can apply it to a multi-antibody epitope-mapping experiment where current approaches are inapplicable due insufficient flexibility. Hence, our approach allows HDX-MS to be applied in more experimental scenarios and reduces the burden on experimentalists to produce excessive replicates. Our approach is implemented in the R-package "hdxstats": https://github.com/ococrook/hdxstats.
\end{abstract}

\section{Introduction}

Probing a protein structure is essential to fully understand its function (Orengo et al., 1999). Protein structures can be perturbed when binding to another protein, small molecule or due

*oliver.crook@stats.ox.ac .uk 
to alterations in their context (such as pH)(Houde et al., 2011; Masson et al., 2017; Sauve et al., 2018). Hydrogen deuterium exchange (HDX) mass spectrometry is one such technique to examine differential context-specific protein structure (Engen, 2009; Chalmers et al., 2011). The guiding hypothesis is that, when a protein is incubated with heavy water, amide hydrogens exchange with deuterium in accordance with their solvent accessibility (Katta and Chait, 1993). Though a number of factors, such as topological flexibility, amino acid content and secondary structure, also affect the process (Konermann et al., 2011; Kammari and Topp, 2020; Jia et al., 2020). By monitoring the kinetics of HDX using bottom-up mass spectrometry, it is possible to discern subtle alterations to a protein's structure (Masson et al., 2017). To mediate possibly complex kinetics the process is usually examined over a time-course. However, this temporal component is rarely used in statistical testing of alterations to the kinetics e.g. (Slysz et al., 2009; Liu et al., 2011; Pascal et al., 2012; Rey et al., 2014; Hourdel et al., 2016; Hageman and Weis, 2019; Lau et al., 2020; Zhang et al., 2020).

A number of approaches have been proposed to detect differences in peptide HDX between samples. These include manual examination of the data, linear models, linear mixed-models and a student's t test amongst others (Slysz et al., 2009; Liu et al., 2011; Pascal et al., 2012; Rey et al., 2014; Hourdel et al., 2016; Hageman and Weis, 2019; Lau et al., 2020; Zhang et al., 2020). The most statistically advanced approach to analyse differential HDX is termed MEMHDX (Hourdel et al., 2016), which introduced random-effects to previous linear modelling approaches (Liu et al., 2011). Models incorporating both fixed effects and random effects are called mixed effects, these model excel at modelling nested variance according to the replicate structure of the data (Schall, 1991). However, they can be difficult to interpret for unseasoned users. Furthermore, MEMHDX suggest to examine $p$-values for magnitude of deuterium change and change in dynamics. This subtly conflates significance with effect size and we suggest to examine a number of effects alongside the $p$-value to accurately interpret the nature of the kinetic changes.

All currently proposed methods avoid explicitly modelling the temporal component of HDX data, which reduces statistical power. In experiments with low samples sizes, such as HDX-MS where only a handful of measurements are made per peptide, maximising power is crucial to infer condition-specific differences. Hence, methodology that models serial correlations will improve power in HDX-MS experiments with low samples sizes. Statistics based on sampled curves, so-called functional data analysis, concerns the analysis of such functions (Ramsay and Silverman, 2007). Here, we turn the physical model of HDX into a statistical test, namely a functional analysis of variance (ANOVA). This approach is simpler to interpret and more powerful than previously proposed methods. The advantages arise because of a reduction in the number of parameters and tests performed, whilst also allowing the model to capture serial correlations (Ramsay and Silverman, 2007). Furthermore, we can exploit repeated measurement across different peptides to estimate a pooled variance. The estimated sample variance for each peptide can then be shrunk towards this pooled variance, resulting in more stable estimates of variance when the sample size is small. This idea is called empirical Bayes and has been highly influential in the analysis of microarrays (Ritchie 
et al., 2015), RNA-seq (Love et al., 2014) and proteomics experiments (Goeminne et al., 2018). We establish this method for HDX data.

Our article is structured as follows. First, we compare a number of methodologies through simulated examples to demonstrate the effects of different methodological choices, replicate structure and number of time-points. We then apply our approach to a number of real-world experiments and find that linear mixed models are unable to control false positives, whilst the t-test is unable to declare any results significant. We then proceed to an epitope mapping experiment for which none of the current methods are applicable. We demonstrate that our approach is able to make quantitative statements in experimental scenarios, where other available methods are not. In particular, our method uncovers significantly altered kinetics in an epitope mapping experiment applied to an HOIP-RBR, for which previous results have only been qualitative (Tsai et al., 2020). Our results support an observation that some single domain antibodies hold HOIP-RBR in a more open conformation (Tsai et al., 2020).

Our approach is made freely available to the community through an R-package "hdxstats": https://github.com/ococrook/hdxstats, which builds on the "QFeatures" package for general quantitative mass-spectrometry (Gatto and Vanderaa, 2021)

\section{Main}

\section{$2.1 \quad$ Simulations}

To obtain a more reliable statistical method for hydrogen-deuterium exchange mass spectrometry (HDX-MS), we developed an empirical Bayes functional model based on a proposed Weibull model for the kinetics (see methods). Our approach can be summarised as fitting a physical model that is blinded to the condition. The model is then refitted separately for each condition. By examining the residual error of each of these two scenarios, we can compute an F-statistic, which is then moderated by pooling information across peptides. The null hypothesis is that the condition-independent model is sufficient to explain the data. Figure 1a shows the fitted model kinetics where there is no difference between the two conditions, whilst panel b shows a situation where the kinetics significantly differ between the two conditions. From these fitted models we can compute an F statistic, high values of the F statistics indicate sufficient evidence to reject the null hypothesis. Such high values are obtained by examining the appropriate F distribution (see methods and Figure 1c). Such an approach avoids testing each time point separately, explicitly incorporates serial correlations and improves power by stabilising variance estimates.

We compare our method to other major approaches (MEMHDX (Hourdel et al., 2016) and t-tests). T-tests are an exact test for the equality of means of two populations. In HDX-MS, this corresponds to application of the t-test pointwise at each measured time point. If the false discovery rate (FDR) for a peptide is lower than some nominal value, say 0.05, we have sufficient evidence to say the HDX kinetics were perturbed at that time point. 
MEMHDX is a linear mixed modelling approach. These approaches are usually applied when multiple measurements are made on the same quantity of interest, which induces a structured variance. In the case of MEMHDX, replicates are encoded as a random effect. If the coefficients of the linear model related to the condition (see methods) are declared significant, then this would be taken as evidence of perturbed HDX kinetics. We note that t-tests are a special case of linear mixed models with one level and random intercepts. Since the t-test and linear mixed models produce many $p$-values per peptide, they are combined to a single $p$-value using the harmonic mean $p$-value (Good, 1958). All methods are corrected for multiple testing using the Benjamini-Hochberg procedure (Benjamini and Hochberg, 1995).

In our simulation study (see methods), we are assessing whether methods can detect known perturbations to the HDX kinetics. If a method declares an FDR of less than 0.05 for a given peptide which has condition-dependent kinetics, then this is called a true positive. Whilst, if the FDR is less than 0.05 but the peptide does not have condition-dependent kinetics then that is defined as a false positive. Hence, methods are assessed using the $\mathrm{F}$ score (not to be confused with the F statistic), which weighs up precision and recall. In all cases, our approach outperforms the other methods except in the last simulation where only $1 \%$ of peptides were simulated to have significant differences, where it performed equally well to the t-test. This performance level indicates that our approach can reliably detect differences even with only a couple of replicates and can handle HDX measurements that are missing at random. The t-test performs poorly because it is difficult to accurately estimate the population standard deviation and thus it is underpowered. MEMHDX performs better than the t-test because it models additional correlations via the random effect but because these correlations are not explicitly parametrised they are less powerful than our functional model. In the next section, we demonstrate that MEMHDX also inflates false positives. 
a

Example with no significant difference

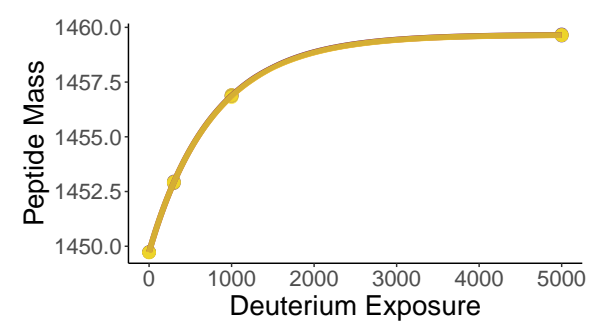

b

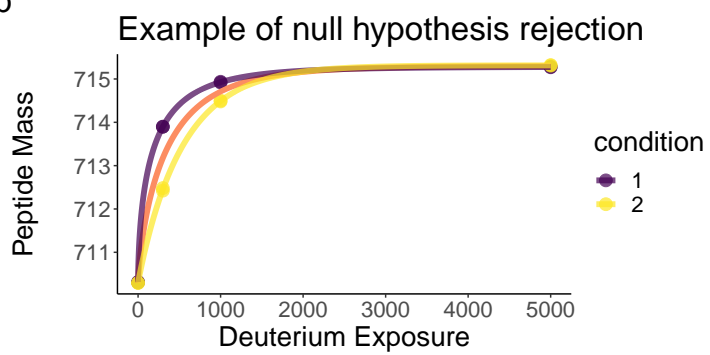

C

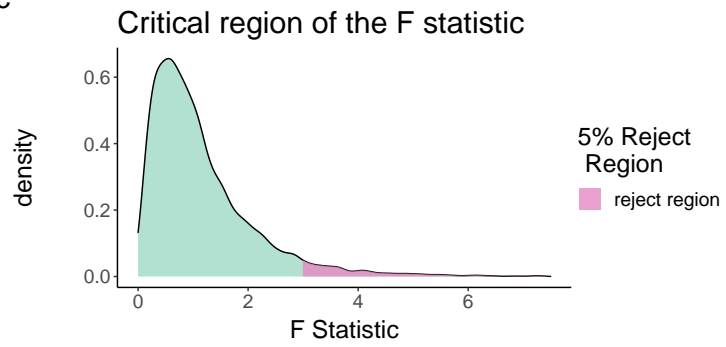

d

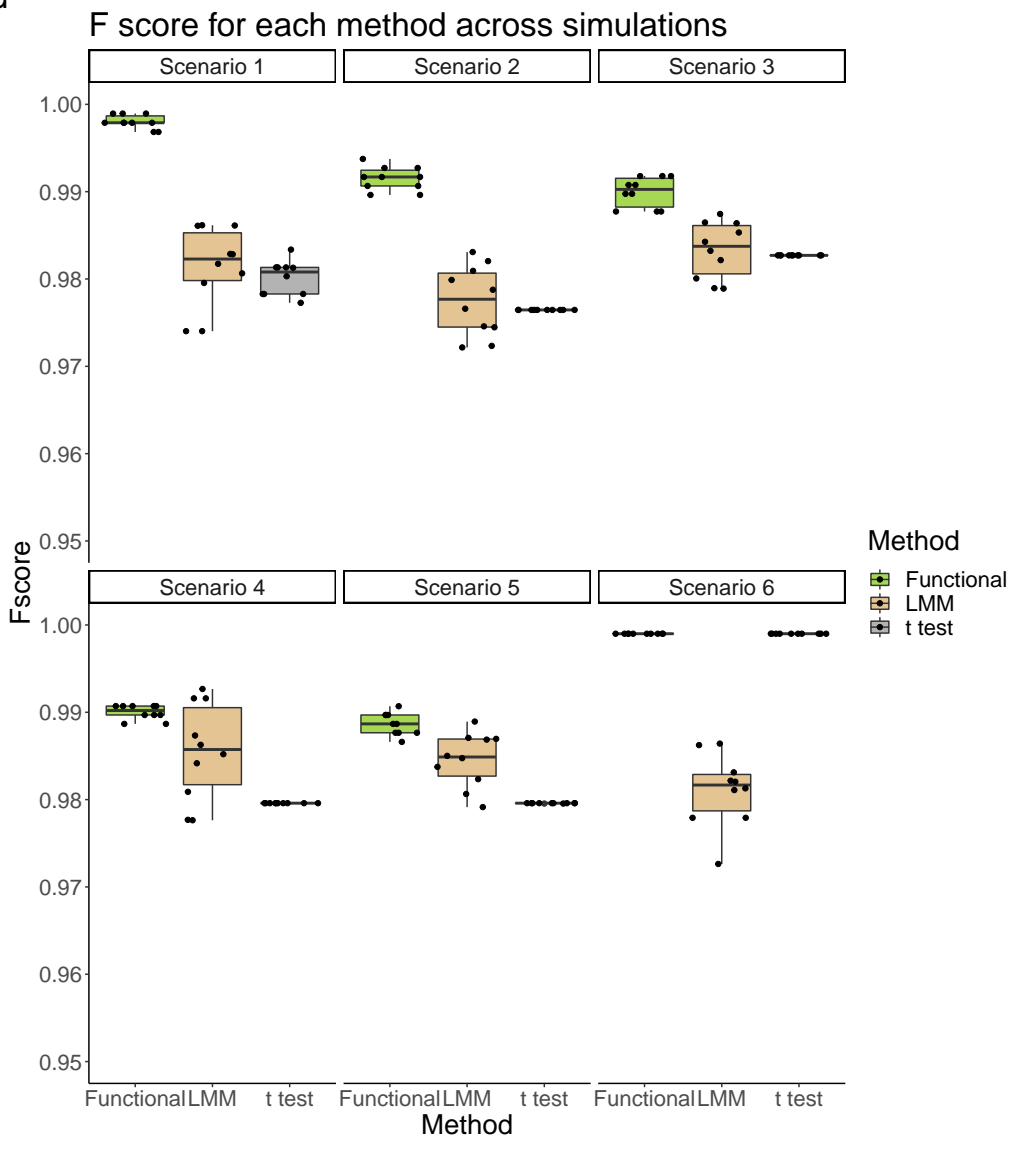

Figure 1: Functional model and performance in simulations. a) Functional models fit where there is no difference between the conditions. b) Functional models fitted to HDX data where there are significant differences c) the $\mathrm{F}$ distribution with $5 \%$ critical region identified in dark green. d) Simulation study for HDX data showing improved performance of our proposed functional method (functional) for linear mixed models (LMM) and the t-test ( $\mathrm{t}$ test). These simulations encompass scenarios with differing numbers of time-points and replicates. We refer the methods for details

\subsection{Applications}

\subsubsection{Structural variant experiment}

We compared each of the approaches in practice. Outside of simulations, true positives are not well defined; however, the methods can be tested on their ability to control false positives. We examined a previously published structural variant experiment, where HDX data on maltose-binding protein (MBP) was generated in seven replicates across four HDX labelling times (Hageman and Weis, 2019). Additional experiments were carried out in triplicate for the W169G (tryptophan residue 169 to glycine) structural variant. Here MBPW169G was spiked into the wild-type MBP sample in 5, 10,15,20,25\% proportions, and a 
further experiment included a 100\% mutant sample. All data were analysed on a Agilent 6530 Q-TOF mass spectrometer and raw spectra processed in HDExaminer.

The seven MBP samples without any structural variant can be used as a null experiment by partitioning the replicates falsely into two conditions. That is three of the samples are labelled condition $A$ and four samples are labelled condition $B$, arbitrarily. We randomly permute the samples labelled $A$ and $B$, six times. We then performed statistical significance testing between the conditions using the three methods previously considered. Since experiments are in fact all replicates, if the FDR is correctly controlled, there should be no peptides declared significant.

From Figure 2 we see that our proposed method and the t-test perform well at avoiding false positives, each generating only one false positive across the six permutation experiments. However, the linear mixed modelling approach generates excessive false positives, with between $6 \%$ and $22 \%$ false positives per experiment. Here, the percentage is of the total peptides measured in the experiment. Hence, we can conclude that the linear mixed modelling approach is too liberal to be reliable in practice.

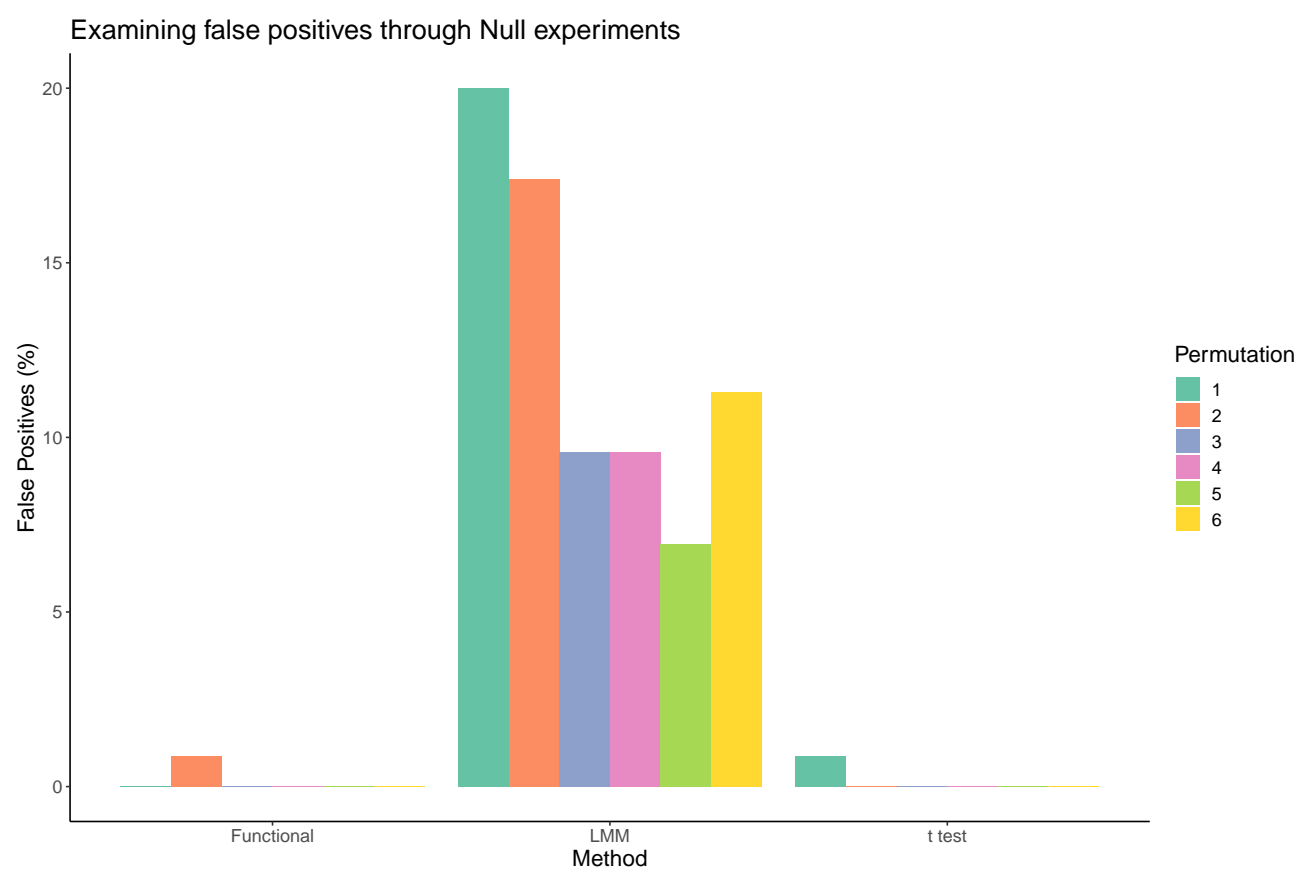

Figure 2: Peformance of statistical methods in null experiments. Our functional approach (functional), linear mixed models (LMM) and the t-test ( $\mathrm{t}$ test) are compared in null experiments.

Our proposed functional statistical approach is built using a parametric physical model allowing us to interpret the statistical significance of our results, beyond simply pairwise differences. We compared wild-type MBP with the $100 \%$ structural variant sample, using all methods. Given there are seven replicates of the wild-type protein, statistical power (and henceforth, simply, power) is not an issue for any of the approaches. However, for most 
experiments, seven replicates is likely to be onerous. Each parameter of our functional model can be interpreted and the magnitude of the parameters can be considered as effects sizes. We are particular interested in the parameters $b$ and $p$ of our functional model because they control the time-dependent kinetics (see methods for a full description). In particular, values of $p>1$ suggest more rapid than exponential exchange of deuterium suggesting that a region has become more exposed to the solvent. Meanwhile, $b$ controls the rate of plateau, such that larger values of $b$ indicate the deuterium uptake plateaus more quickly. The parameter $a$ models the plateau itself, whilst $d$ models the intercept. A forest plot can be used to simultaneously visualise several possible effects that we might be interested in to improve the interpretation of the results. Figure 3 shows two peptides with overlapping residues and their fitted models. In both cases the kinetics are significantly different between the WT and structural variant $\left(\mathrm{FDR}<10^{-8}\right)$ using our empirical Bayes F-test. Panels $\mathrm{c}$ and $\mathrm{d}$ of this figure show that the pairwise differences at each time-point are different from 0 . The parameters $d$ and $b$ only display small changes, whilst $a$, which models the curves' plateau, is significantly different. This suggests some residues for these peptides have become accessible due to the mutation compared with the WT protein. We can also see that $p \approx 1.5$ for peptide IAYPIAVEA [129-138] and $p \approx 1.8$ for YPIAVEAL [131-139] in the W169G variant, whilst for the WT $p \approx 1$ in both cases. This suggest more rapid than exponential exchange in deuterium for the mutant and further evidence that this region has become more solvent accessible and/or has fewer hydrogen bonds. The concerted behaviour between the two peptides adds further support to this hypothesis. These peptides are localised to a buried beta-strand suggesting the structural variant has a large impact on the protein's structure (figure $3 \mathrm{e}$ ). 
We then assessed our method on its ability to detect subtle differences in HDX experiments. We took the $10 \%$ and $15 \%$ structural variant samples from the structural variant experiments on MBP. We applied our functional model to the data and found that 12 peptides had an adjusted $p$-value smaller than 0.05. Three examples are plotted in figure 4 panels a,b,c with the remainder plotted in the supplement. Figure 4 panel d shows the forest plot corresponding to the peptide in figure panel a. Here, the difference is visually subtle but our functional method identifies a difference between the two samples. Indeed, at all time points, the deuterium incorporation for the $10 \%$ sample is lower than that of the $15 \%$ structural variant sample. However, for three out the four samples, the confidence intervals in this difference overlapped with 0 (see table 1). The power of functional methods is that they can identify the consistency in an effect across time points, allowing us to identify significant changes that are consistent but not necessarily significant at any time point individually. This finding is reinforced with an overlapping peptide demonstrating concordant behaviour (figure 4 panel $\mathrm{b}$ and $\mathrm{g}$ ). The t-test fails to find any significant differences at level 0.05 and the histogram of adjusted $p$-values is shown in figure 4e. The lack of uniformity and peaking of values toward 0 suggests low power, whilst the expected trend is seen for the functional method in figure 4f. Even deuterium differences that are apparent are not detected by the t-test (see 4c). Our functional models ability to detect differences whilst controlling falsepositives in both simulations and experiments suggests it is a more appropriate method for HDX-MS.

\begin{tabular}{rrrrll}
\hline & Estimate & confL & confU & Time point & condition \\
\hline 1 & -0.17 & -0.31 & -0.03 & 30 & Change in Deuterium Uptake \\
2 & -0.03 & -0.09 & 0.02 & 240 & Change in Deuterium Uptake \\
3 & -0.02 & -0.14 & 0.11 & 1800 & Change in Deuterium Uptake \\
4 & -0.01 & -0.13 & 0.11 & 14400 & Change in Deuterium Uptake \\
\hline
\end{tabular}

Table 1: Difference between deuterum incoperation for peptide DIKDVGVDNAGAKAGLTF in the $10 \%$ and $15 \%$ structural variant experiments. Central estimates (mean) are reported alongside the $95 \%$ confidence intervals. The lower (upper) tail of the confidence interval is given by confL (confU). 

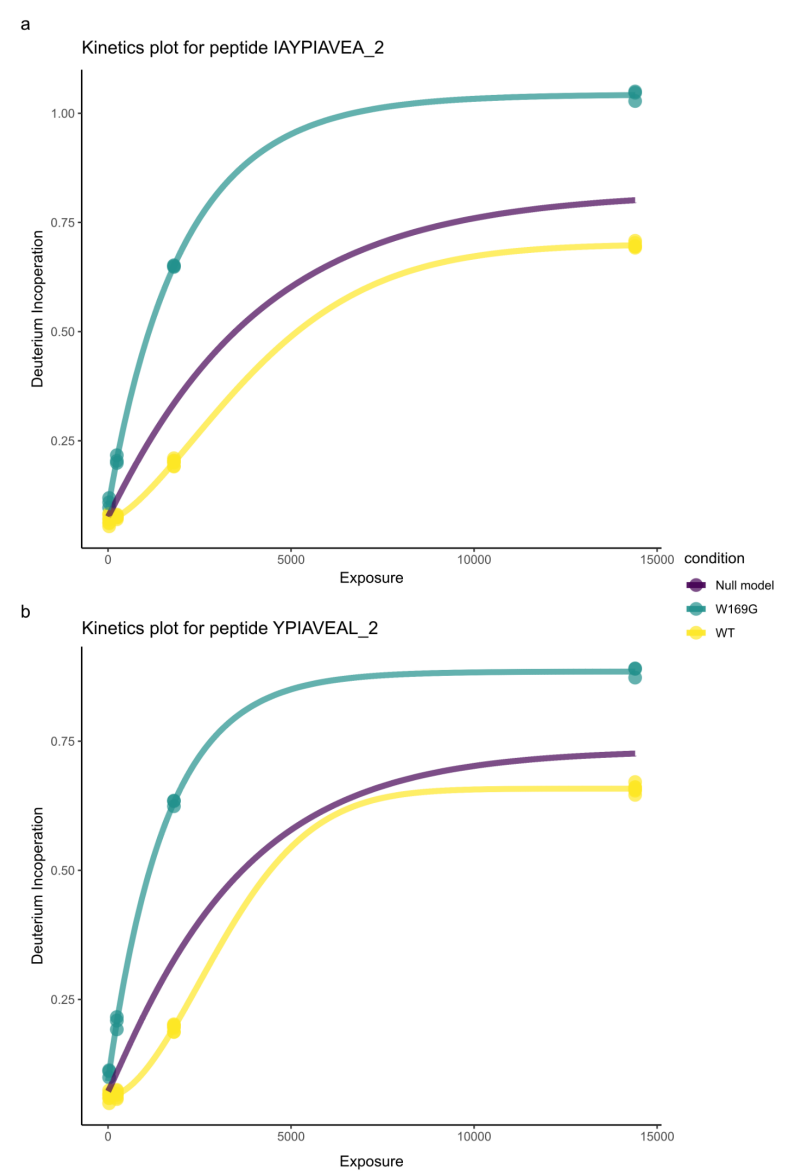

$\mathrm{e}$

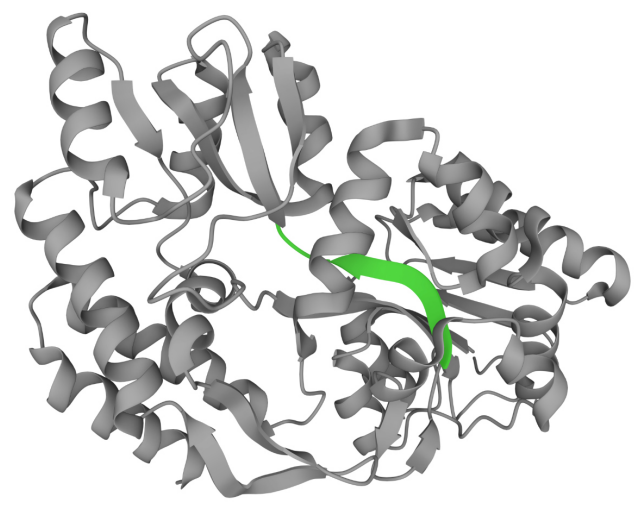

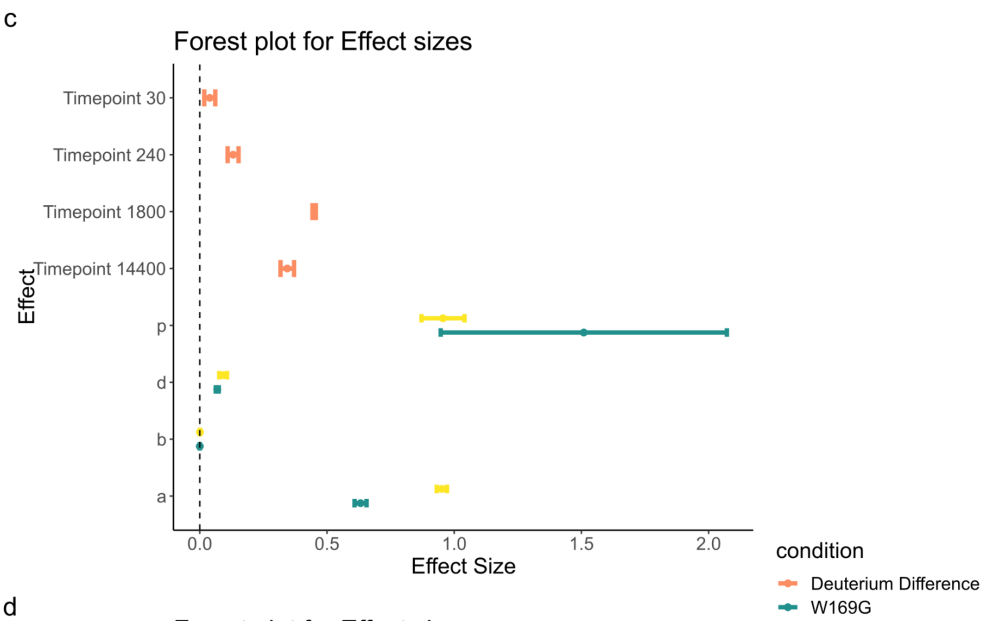

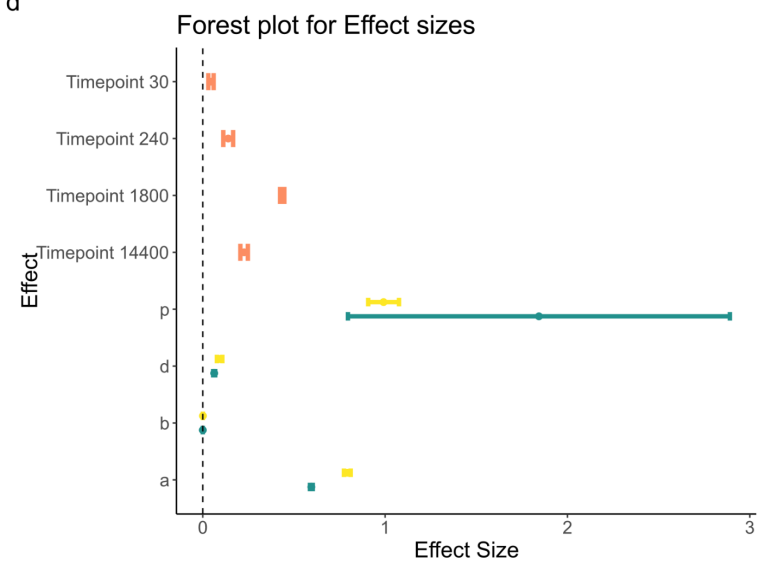

W169G

Figure 3: Functional models are interpretable. (a) Deuterium kinetics for peptide IAYPIAVEA [119-138] in charge state two. Proportion of deuterium incorporated is plotted against solvent exposure time in seconds. Conditions are coloured with the null model in purple. (b) same as for (a) but for peptide YPIAVEAL [131-139] in charge state one. (c) Forest plot for effect sizes corresponding to peptide in IAYPIAVEA [129-138]. (d) Forest plot for effect sizes corresponding to peptide in YPIAVEAL [131-139]. (e) MBP (PDB: 1OMP) with peptides highlighted in green YPIAVEAL [131-139](left) IAYPIAVEA [129-138] (right) 

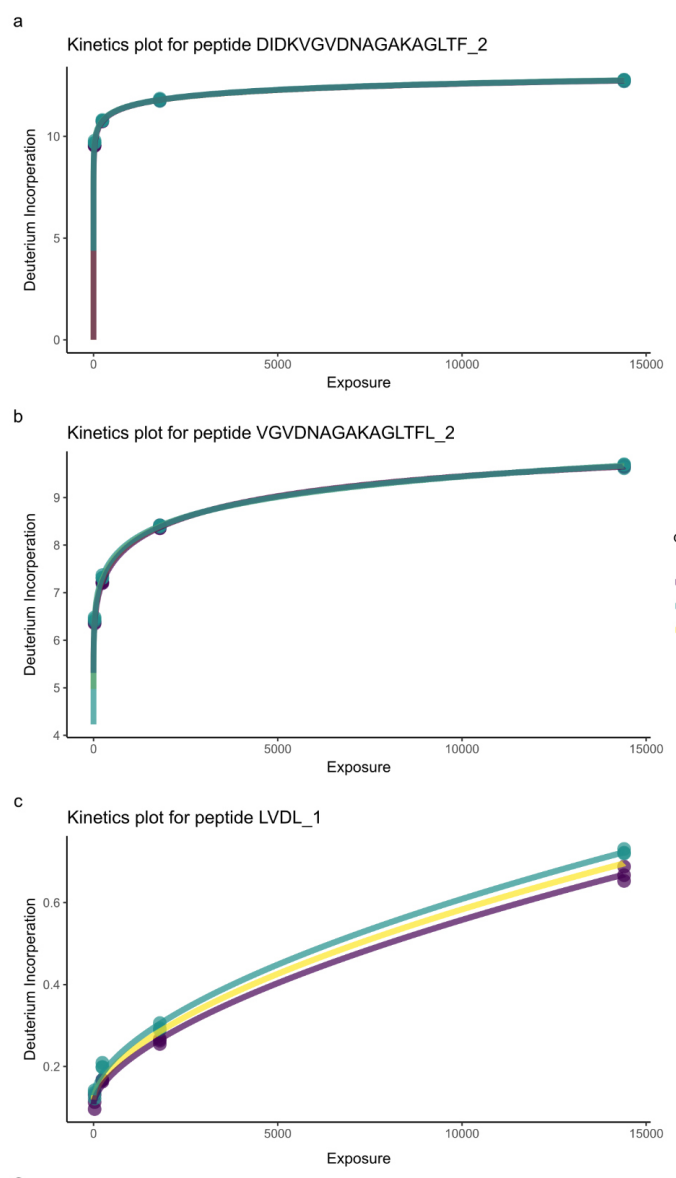

G

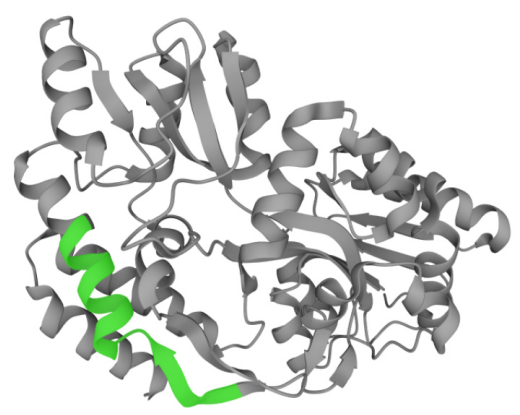

d

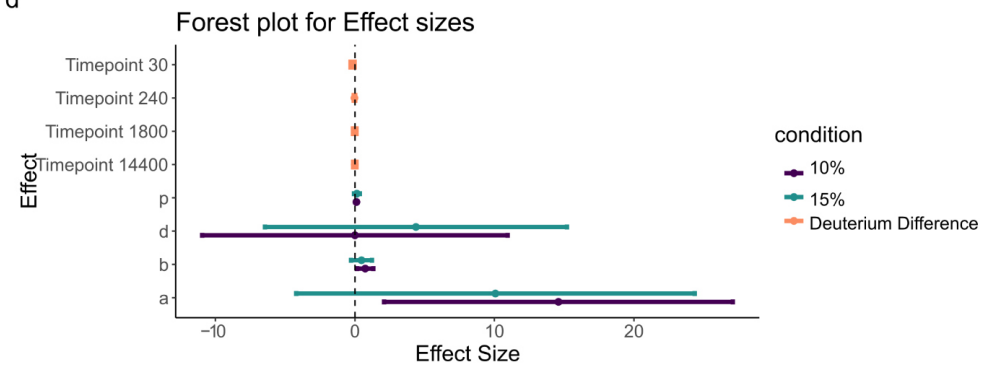

$\mathrm{e}$

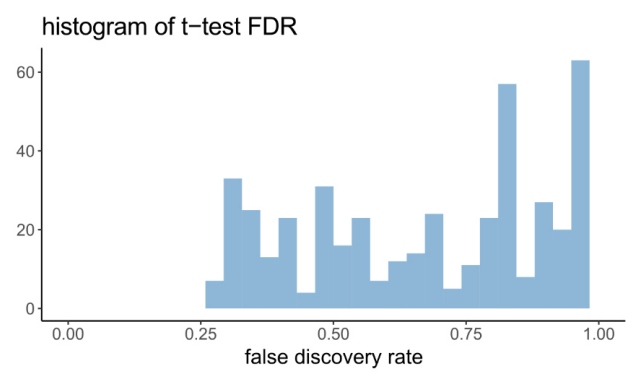

f
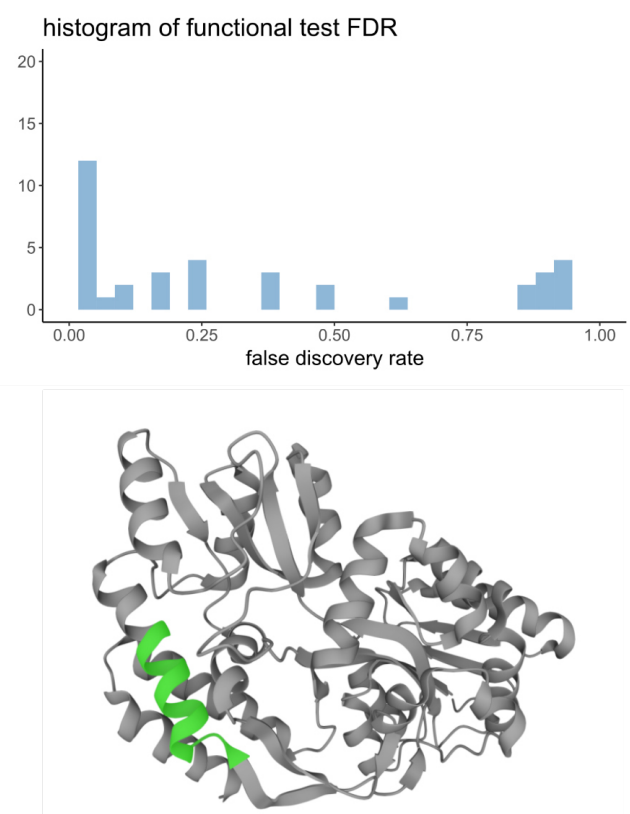

Figure 4: Functional models can identify subtle, consistent differences. (a) Deuterium kinetics for peptide DIKDVGVDNAGAKAKAGLTF [203-220] in charge state 3. Deuterium incorporation is plotted against solvent exposure time in seconds. Conditions are coloured with the null model in yellow. (b) same as for (a) but for peptide VGVDNAGAKAGLTFL [207-220] in charge state 2. (c) as for (a) but for peptide LVDL [221-224] in charge state 1 (d) Forest plot for effect sizes corresponding to peptide in (a). (E,F) Histogram of adusted $p$-values for the t-test and functional method, correspondingly. (G) MBP (PDB: 1OMP) with DIKDVGVDNAGAKAKAGLTF (left) and VGVDNAGAKAGLTFL (right) highlighted in green. 


\subsubsection{Epitope mapping of HOIP-RBR using HDX-MS}

In this section, we explore an application of HDX-MS to epitope mapping of HOIP-RBR. HOIP is an E3 ubiquitin-protein ligase which conjugates linear polyubiquitin chains and plays a role in immune signalling (Kirisako et al., 2006; Gerlach et al., 2011; Spratt et al., 2014). Usually, binding epitopes are identified by "protection"; that is, surface amides that incorporate deuterium more slowly as they are shielded from the solvent. Tsai et al. (2020) performed HDX-MS experiments for HOIP-RBR upon single domain antibody (dAb) complexation and in APO state. Mass-spectrometry was performed using a Waters Synapt G2-Si instrument and raw data was processed using DynamX. HDX-MS measurements were taken at 0,30 and 300 seconds post exposure to heavy water, for thirteen dAbs at different molar concentrations. Only a single replicate measurement was taken in each state so that more measurements of different dAbs could be made. However, this renders the t-test inapplicable because we cannot compute a within group variance and linear mixed-models (MEMHDX) are inapplicable because there is not a nested replicate structure. However, it is still possible to apply our proposed functional method.

To avoid over-fitting, we fix $b=0.5, p=1, d=0$ in our Weibull model, reducing the complexity of our model to a single degree of freedom in the null case. This greatly reduces the flexibility of our model, but in return we can apply rigorous statistical testing. These parameter choices roughly correspond to an assumption that $80 \%$ of deuterium will be incorporated within 30 seconds and the kinetics will plateau by 300 seconds.

For brevity we focus on dAb25 from the study of Tsai et al. (2020), because they observed non-standard HDX behaviour for this complex (the remainder are shown in the supplement). We applied our functional method as detailed in the methods, with the alterations described in the previous paragraph. We identified eight peptides for which the deuterium kinetic were altered (adjusted $p$-value $<0.05$ ). six of these peptides displayed non-classical behaviour with increased deuterium incorporation on $\mathrm{dAb}$ binding (see figure 5). Three of these peptides overlap with each other and are contained within the helix-turn-helix (linker) region of HOIP (top row figure 5), whilst the other three also overlap and are contained in the RING2 region of HOIP (middle row figure 5)). The remaining two peptides also overlap with each other and are contained within the IBR (in-between ring) region of HOIP (final row figure 5). This suggests that the epitope for dAb25 is contained with the IBR region and this binding holds HOIP in a more open conformation allowing increased solvent exposure; hence, more deuterium exchange is possible.

To provide the spatial context for these changes in deuterium kinetics, we plotted a Manhattan plot; that is, peptides plotted against $-\log _{10}$ ( $p$-value) (see Figure 6 ). This helps us to simultaneously visualise protein domain regions and (de)protected regions, as described in the previous paragraph. Whilst examining this plot, we also notice tendencies for $p$-values to cluster, suggesting correlations in the spatial axis of HDX data. This is expected, since these peptides either physically overlap or come from the same protein domain. Furthermore, we notice some of these clustered $p$-values fall just below the significance threshold 
suggesting power could be boosted by modelling correlations in this dimension as part of future work. Results for the remaining peptides and a residue-level analysis can be found in the supplementary materials.
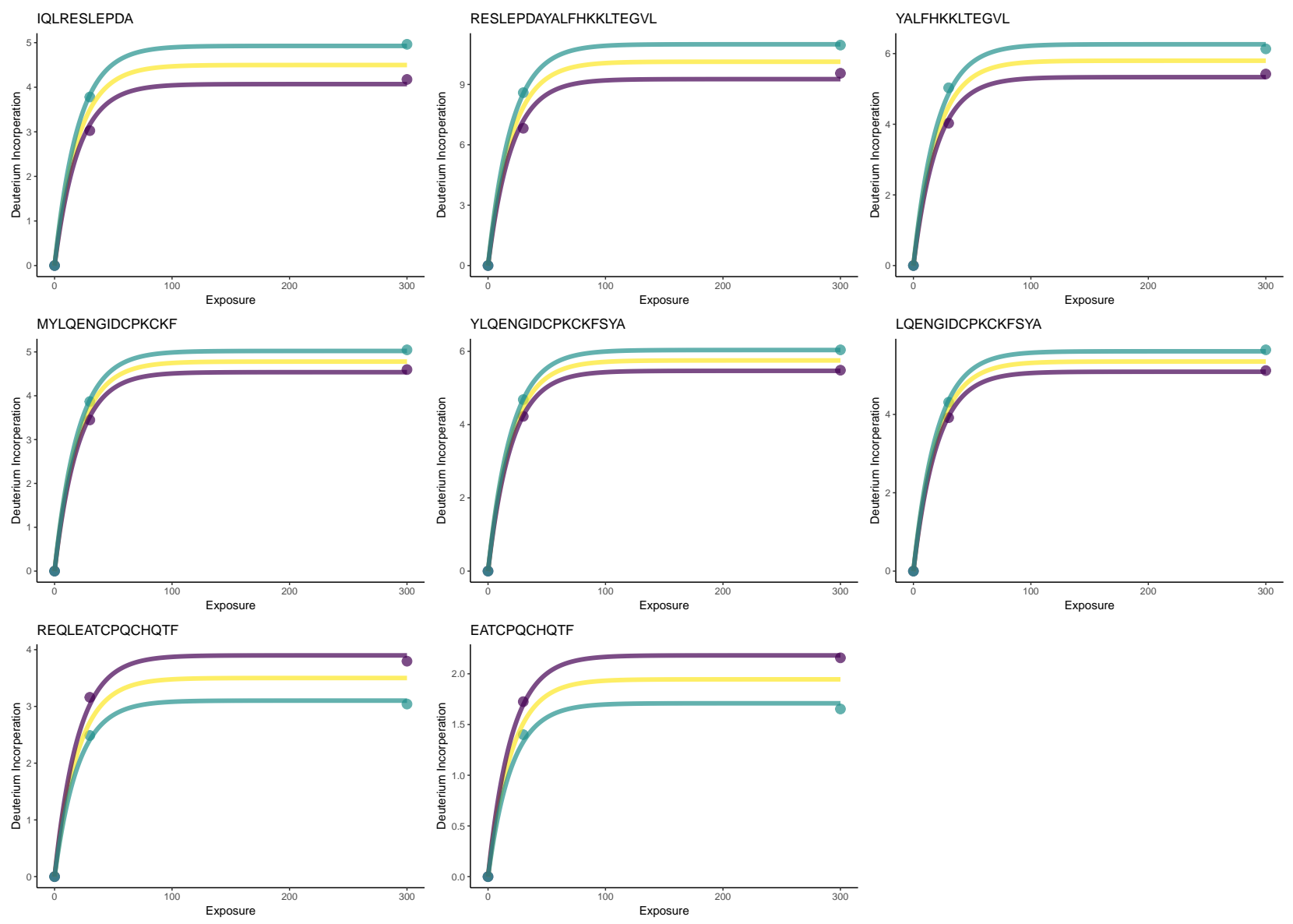

Figure 5: Functional model identifies protected and unprotected region of HOIPRBR in complexation with dAb 25. The top row contains kinetic plots for peptides in the helix-turn-helix region of HOIP; the middle row contains kinetic plots for peptides in the RING2 region of HOIP; the final row contains proteins in the IBR region of HOIP. Deuterium incorporation is plotted in units of Daltons and Exposure to heavy water in seconds. Yellow lines indicate the null model, whilst green and purple indicates the alternative model. 


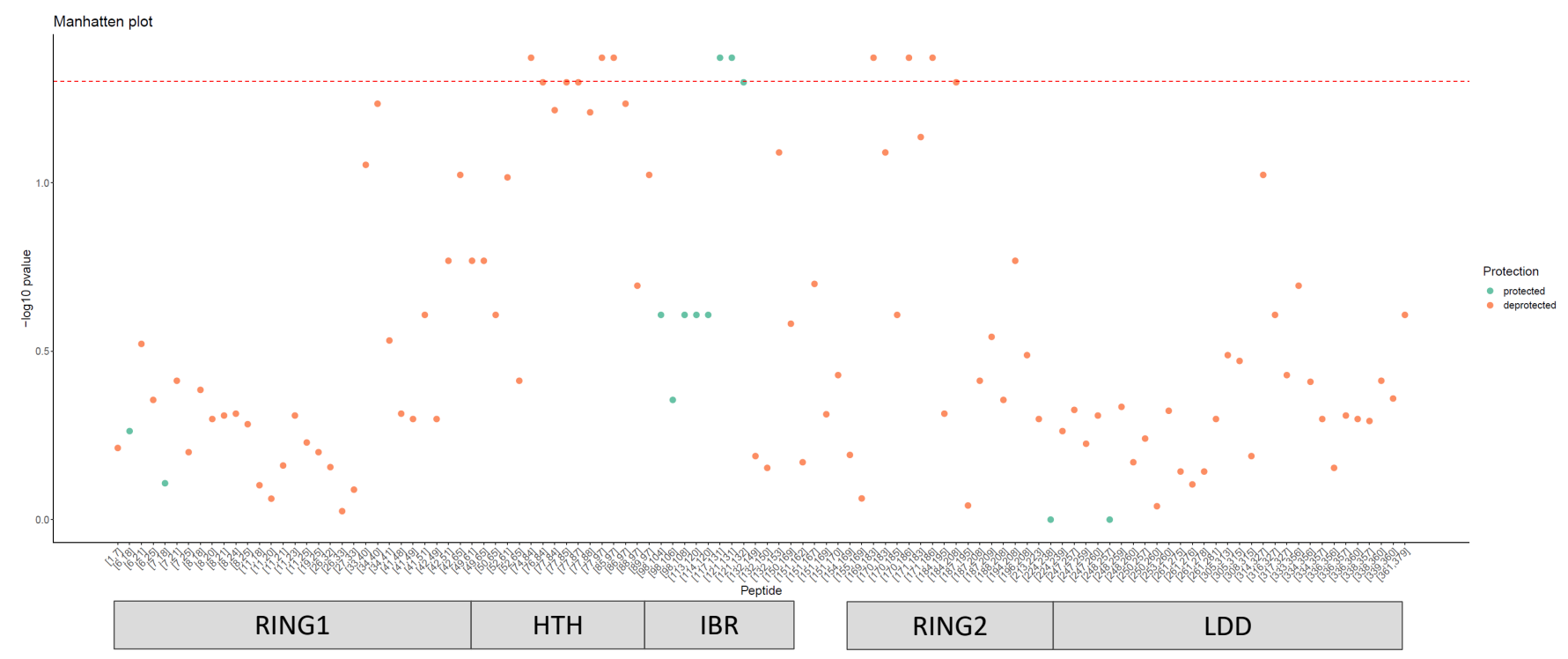

Figure 6: Manhattan plots provide spatial context of deuterium chances for HOIPRBR-dAb25. A Manhattan plot with peptides described as the amino acid (AA) segments of the protein sequence plotted against the $-\log _{10}(p$-value). Protein domain annotations are provided in the bar below (Tsai et al., 2020). 


\section{Discussion}

We have presented an empirical Bayes functional data analysis approach for HDX-MS data. Our model explicitly incorporates the temporal component of these data, which boosts power and interpretation. Furthermore, we developed an empirical Bayes testing approach to stabilise variance estimates across the peptides in the experiment. The resulting methodology is more powerful than previous linear model-based approaches, as demonstrated by our simulation study. These earlier approaches lack power because they do look explicitly incorporate the temporal component nor borrow information across peptides.

We made an empirical comparison of the approaches in an application to structural variant data. This analysis concluded that the mixed-modelling approach was unable to control the false discovery rate, whilst our approach and the t-test were able to control it. However, application to a case with subtle differences demonstrated that the t-test was unable to declare any peptides significant. Hence, our approach controls false positives whilst providing peptides which can be followed up.

Having demonstrated the empirical statistical properties of our method, we applied our approach to a case-study of multi-antibody epitope mapping of HOIP-RBR. Current approaches are not able to assess significance in these experiment because of their stringent assumptions. However, our empirical Bayes functional method is applicable and was able to find significant differential HDX kinetics. Thus, we are able to identify the binding epitopes and allosteric effects of the single domain antibodies on HOIP-RBR.

Our approach has a number of limitations. Firstly, we do not model correlations in the spatial domain of HDX data - that is between overlapping peptides. This manifests as clustering of $p$-values for overlapped peptides. Secondly, we do not model correlations in our multi-antibody study across the different antibody experiments. Joint modelling across related experiments is likely to boost power and interpretation further. Finally, our analysis works with centroided HDX spectra, we anticipate further improvements by working with raw spectra and identification confidences directly.

\section{Methods}

\subsection{Preliminaries}

In hydrogen deuterium exchange mass spectrometry, we observe isotope distributions for $i=1, \ldots, n$ peptides at different exposure times $t_{1}, . ., t_{m}$ to heavy water $\left(\mathrm{D}_{2} \mathrm{O}\right)$. The isotope distributions are a set of $\frac{m}{z}$-Intensity pairs revealing the relative intensities of each peptide isotope. These isotope distributions are frequently summarised into a centroid via an intensity weighted mean of the $\frac{m}{z}$, which we write as $\frac{\bar{m}}{z}$. Since deuterium is heavier than hydrogen, deuterium interoperation leads to positive shifts in $\frac{m}{z}$ and monitoring this change over time and with respect to the state is the standard usage of HDX-MS. In most scenarios data are replicated, so we observe replicates $r=1, \ldots, R$ and, potentially, a number of conditions 
denoted $c=1, . ., C$. For example, binding to antibody is an example of a condition. The observations are then

$$
y_{i c r}(t)=M(t)=\frac{\bar{m}}{z}(t) \times z-z
$$

where $z$ denotes the charge of the precursor ion. Some practitioners normalise with respect to the initial mass $M(0)$, though this is unnecessary and assumes that mass errors are similar over time. This normalisation is an assumption of homoscedasticity, which is unlikely to hold in general because as deuterium is incorporated the isotope distribution undergoes isotopic expansion and so $M(t)$ will have different errors for different values of $t$. If we wish to avoid this normalisation, we can include an offset term in our proposed model (see below).

\subsection{Proposed Method}

In this section, we describe the functional model we use to model hydrogen deuterium exchange. The kinetics of HDX follow a well-appreciated logistic-type model. Typically, peptides rapidly incorporate deuterium and plateau at maximum incorporation. Mathematically, the proposed model takes the following form

$$
\mu(t)=a(1-\exp (-b t))+d
$$

Each parameter is interpreted as follows. Firstly, $d$ denotes the mass at time 0, but note this is inferred so the uncertainty in the value is captured unlike when normalising by $M(0) . b$ controls the time dependent kinetics, such that larger values of $b$ denote a more rapid increase in mass (and hence deuterium incorporation). Whilst a controls the plateau of the model representing maximum incorporation. It is also useful to consider a slight modification to the above model, in which a Weibull-type model is used:

$$
\mu(t)=a\left(1-\exp \left(-b t^{p}\right)\right)+d
$$

The additional parameter $p$ models additional effects with respect to the temporal kinetics. Sometimes, values of $p$ are greater than 1, suggesting more rapid than exponential incorporations at smaller times, and plateauing more quickly than exponential on longer time scales. Mathematically, $p$ allows some flexibility in the inflexion of the kinetics.

\subsection{Two-sample test}

The proposed model can be turned into a formal test, using tools from functional data analysis. We first suppose that there is no difference in HDX kinetics for a peptide between two conditions. In such a case, a single function would describe the HDX kinetics regardless of the condition. This constituents the null hypothesis, whilst the alternative is that there is a difference in HDX kinetics. In this case, independent condition-specific models would better describe the data. To formalise this, for each peptide $i$, we would fit the following model

$$
\mu_{i c}(t)=a_{i c}\left(1-\exp \left(-b_{i c} t^{p_{i c}}\right)\right)+d_{i c}
$$


and correspondingly compute the residual sum of squares under the null (subscript 0) and alternative (subscript 1) hypothesis:

$$
\begin{aligned}
\mathrm{RSS}_{0, i} & =\sum_{c, r, t}\left(y_{i c r t}-\mu_{i 0 t}\right)^{2} \\
\mathrm{RSS}_{1, i} & =\sum_{c, r, t}\left(y_{i c r t}-\mu_{i c t}\right)^{2}
\end{aligned}
$$

These equation describe the squared deviation of the observed data from the fitted mean functions. These quantities will be small if the model is a good fit. The relative plausibility of the two models can be described using the $F$-statistic:

$$
F_{i}=\frac{d_{i, 2}}{d_{i, 1}} \frac{\mathrm{RSS}_{0, i}-\mathrm{RSS}_{1, i}}{\mathrm{RSS}_{1, i}},
$$

which intuitively weighs up the relative fits of the null and alternative models. The values $d_{i, 1}$ and $d_{i, 2}$ represent the degrees of freedom of the corresponding $F$-distribution. $d_{i, 1}$ is given by $p_{2}-p_{1}$, that is the difference between the number of parameters in the alternative model and the null model, for our approach $p_{1}=3$ and $p_{2}=6$ or $p_{1}=4$ and $p_{2}=8$ if the Weibull model is used. Whilst, $d_{i, 2}=n_{i}-p_{2}$, where $n_{i}$ is the number of observations of peptide $i$ across the conditions. Finally, $p$-values are obtained from the corresponding $F$-test:

$$
F_{i} \sim_{H_{0}} F\left(d_{i, 1}, d_{i, 2}\right)
$$

Given that we are performing $n$ tests, multiple testing correction should be performed, typically the Benjamini-Höchberg precdure is recommended (Benjamini and Hochberg, 1995).

\subsection{Effect sizes}

In linear models, effect sizes are given by the values of the coefficients of the appropriate covariates. For functional models, there are number of possible effect sizes. The appropriate effect size is best chosen based on the question of interest. We describe some of the possible effects that can be extracted from the model:

1. Differences in initial mass:

This quantity is described by extracting $d$ and its confidence intervals from the models.

2. Difference in maximum uptake:

This quantity is described by extracting $a+d$ and its confidence intervals from the models.

3. Difference in rate kinetics:

This quantity is described by extracting $b$ as well as $p$ and their confidence intervals from the models. 
4. The global difference between the two models.

This quantity is given by the maximum difference between the condition-specific curves:

$$
\Delta_{i, \max }=\sup _{t}\left|\mu_{i 1}(t)-\mu_{i 2}(t)\right|
$$

or the integral

$$
\Delta_{i, i n t}=\int_{t}\left|\mu_{i 1}(t)-\mu_{i 2}(t)\right| \mathrm{d} t .
$$

5. Local differences between models; that is, an effect at specific time $t_{*}$.

This quantity is given by the difference between the condition-specific curves at that time:

$$
\Delta_{i, t_{*}}=\mu_{i 1}\left(t_{*}\right)-\mu_{i 2}\left(t_{*}\right)
$$

\subsection{Empirical Bayes}

Estimates derived from a small number of replicates and time-points can lead to unstable inferences, as is typically observed in microarray (Ritchie et al., 2015) and RNA-seq experiments (Love et al., 2014). To improve stability, and hence power, we propose an empirical Bayes extension to our model. The idea is to shrink estimates of the sample variance towards a pooled estimate. This constituents a bias-variance trade-off, where we trade a small amount of bias for increased precision. Following Smyth (2004), inference can then be formulated using a so-called moderated $F$-statistic. To elaborate, let $s_{i}^{2}=\frac{R S S_{i, 1}}{d_{i, 2}}$, we can then use this set of variances to identify a global $s_{0}^{2}$ and shrink our estimate $s_{i}^{2}$ towards $s_{0}^{2}$. We assume true variances $\sigma_{i}^{2}$ are drawn from the following scaled inverse $\chi^{2}$ distribution:

$$
\frac{1}{\sigma_{i}^{2}} \sim \frac{1}{d_{0} s_{0}^{2}} \chi^{2}
$$

It can be shown that (Smyth, 2004), the expected value of the posterior of $\tilde{s}_{i}^{2}$ is

$$
\tilde{s}_{i}^{2}=\frac{d_{0} s_{0}^{2}+d_{2} s_{i}^{2}}{d_{0}+d_{2}},
$$

where the hyperparameters $d_{0}$ and $s_{0}^{2}$ are computed by fitting the following scaled $F$ distribution $s_{i}^{2} \sim s_{0}^{2} F\left(d_{2}, d_{0}\right)$. Hence, we compute the moderated $F$-statistic with

$$
\tilde{F}=\frac{\operatorname{RSS}_{0, i}-\mathrm{RSS}_{1, i}}{\tilde{s}_{i}^{2} d_{i, 1}} .
$$

\subsection{Functional ANOVA}

Our model takes the form of a functional analysis of variance (ANOVA). As a result, it allows for covariate based experimental designs with multiples levels. One such example would be an analysis based on a set of antibodies or different concentrations of small molecules. 


\subsection{Linear mixed-effects models}

In this section, we summarise the previous methodology for significance testing for hydrogen deuterium exchange mass spectrometry that explicitly includes temporal components. The approach, using mixed effects models, is an extension of a previous linear modelling approach (Hourdel et al., 2016; Liu et al., 2011). In our notation, each set of peptide observations is modelled as:

$$
y_{i c r}(t)=\beta_{i t} x_{t}+\beta_{i c} x_{c}+\beta_{t c} x_{t} x_{c}+u_{r} w_{r}+\epsilon,
$$

where $\beta_{i t}, \beta_{i c}$ and $\beta_{t c}$ denote the coefficients of the fixed effects for time, condition and their interaction between time and condition for peptide $i$. The replicates are considered as random effects $u_{r}$. Random effects attempt to model the additional structured variance corresponding to a particular covariate. We note that in this interpretation that time is considered as a factor rather than a continuous quantity. That is, permuting the ordering of time does not change the model and the explicit correlation induced by the temporal dimension is not modelled. In the case that time is interpreted as a continuous quantity, the model becomes

$$
y_{i c r}(t)=\beta_{i t} t+\beta_{i c} x_{c}+\beta_{t c} t x_{c}+u_{r} w_{r}+\epsilon,
$$

however HDX data are rarely linear in time and so the data have to be linearised. This can be performed by transforming time according to a shifted $\log$ transform: $t \rightarrow \log (t+\delta)$. Where $\delta$ is chosen to avoid taking the $\log$ of 0 . Though this approach reduces the number of tests performed and models the temporal dimension it is not recommend as it can lead to uncontrolled $p$-values and unstable parameter estimates.

\subsection{Simulations}

This section describes our proposed simulation study. We begin by sampling, uniformly at random, the length of the peptide between 5 and 25 . The sampled number is the number of amino acids in the peptide and we sample that number of amino acids from the 20 canonical amino acids with replacement. We then define time points at which to obtain data: $T=\left\{t_{1}, \ldots, t_{m}\right\}$, with $t_{1}=0$ and $t_{i}<t_{j}$ for $i<j$. For time $t_{1}$, we simulate the undeuterated isotope distribution using a binomial modal. For a subsequent time point $t_{i}$ we sample the percentage incorporation by first sampling from a $m$ - 1-variate Dirichlet distribution with concentration parameter $\alpha$, where $\alpha_{i}=20 /(i-1)$. From this we obtain a vector $\pi$ which sums to 1 . We use the cumulative distribution of $\pi$ as the schedule of incorporations. That is the incorporation at $t_{i} D_{i}=\sum_{r=1}^{i-1} \pi_{r}$ for $i>1$. This ensure that incorporation is non-decreasing in time. To simulate the effect of a condition, for each time point, we sample an indicator $z_{t_{i}} \in\{0,1\}$ such that the $p\left(z_{t_{i}}=0\right)=0.95$. If $z_{t_{i}}=1$, then we re-sample the incorporation amount and continue on the simulation process. This ensures that roughly $95 \%$ of the scenarios have no effect with respect to the condition. A binomial model is used to generate deuterated spectra, where the exchangeable hydrogens are randomly replaced with deuterium according to the incoperation percentage. The isotope 
distribution simulations are repeated $R$ times to allow for replicates. Centroids summarising the average peptide mass are then computed from the isotope distribution. The centroids are then further corrupted by Gaussian noise, using $\mathcal{N}(0,0.05)$. In all cases we simulate 500 measured peptides. We perform simulation scenarios as follows:

- (Scenario 1) 4 time points, 3 replicates and 2 conditions

- (Scenario 2) 4 time points, 2 replicates and 2 conditions

- (Scenario 3) 5 time points, 2 replicates and 2 conditions

- (Scenario 4) 6 time points, 2 replicates and 2 conditions

- (Scenario 5) 6 time points, 2 replicates, 2 conditions and 5\% missing values

- (Scenario 6) 6 time points, 2 replicates, 2 conditions, $5 \%$ missing values, $p\left(z_{t_{i}}=0\right)=$ 0.99

All simulations are performed 10 times and the distributions compared.

\subsection{Performance metrics}

We use the F-score to assess the performance of the different statistical methods. The Fscore is the harmonic mean of the precision and recall. Precision is defined as $\frac{t p}{t p+f p}$ and recall is $\frac{t p}{t p+f n}$, where $t p$ is true positives, $f p$ is false positives and $f n$ is false negatives. In words, the F-score weighs how many of the selected peptides are relevant and how many of the relevant peptide are selected.

\subsection{Implementation}

The Weibull-type model is implemented using the Levenberg-Marquardt algorithm, an iterative procedure that interpolates between the Gauss-Newton algorithm and gradient descent (Marquardt, 1963). The parameters are all constrained to be non-negative and the algorithm ends after 500 iterations or the difference between successive sum of square residuals is less than $10^{-8}$.

\subsection{Residue-level analysis}

For visualisation purposes, we propose a residue-level analysis in the following manner. For each residue $j=1, \ldots, J$, where $J$ is the total amino acids in the protein, we have a set of $p$-values $j_{p}$, for $p=1, . ., P_{j}$ where $P_{j}$ is the sequence coverage at that amino acid. A residue level $p$-value for residue $j$, for visualisation purposes, is taken as the harmonic mean of the $p$-values $\left\{j_{1}, \ldots, j_{P_{j}}\right\}$. The primary visualisation is a heatmap of the $-\log _{10}$ of this computed value. 


\section{Acknowledgements}

We thank Nathan Gittens for critical reading of the manuscript. OMC acknowledges funding from GSK and a Todd-Bird Junior Research Fellowship from New College Oxford. CWC is an employee of GSK.

\section{Data availability}

Data reproduce the figures is provided in the supplementary material. Experimental data are available from the original manuscripts.

\section{Code availability}

Code is available as part of the R-package "hdxstats": https://github.com/ococrook/ hdxstats.

\section{References}

Benjamini, Y. et al. (1995). Controlling the false discovery rate: a practical and powerful approach to multiple testing. Journal of the Royal statistical society: series B (Methodological), 57(1), 289-300.

Chalmers, M. J. et al. (2011). Differential hydrogen/deuterium exchange mass spectrometry analysis of protein-ligand interactions. Expert review of proteomics, 8(1), 43-59.

Engen, J. R. (2009). Analysis of protein conformation and dynamics by hydrogen/deuterium exchange ms.

Gatto, L. et al. (2021). QFeatures: Quantitative features for mass spectrometry data. R package version 1.3.7.

Gerlach, B. et al. (2011). Linear ubiquitination prevents inflammation and regulates immune signalling. Nature, 471(7340), 591-596.

Goeminne, L. J. et al. (2018). Experimental design and data-analysis in label-free quantitative lc/ms proteomics: A tutorial with msqrob. Journal of proteomics, 171, 23-36.

Good, I. J. (1958). Significance tests in parallel and in series. Journal of the American Statistical Association, 53(284), 799-813.

Hageman, T. S. et al. (2019). Reliable identification of significant differences in differential hydrogen exchange-mass spectrometry measurements using a hybrid significance testing approach. Analytical chemistry, 91(13), 8008-8016. 
Houde, D. et al. (2011). The utility of hydrogen/deuterium exchange mass spectrometry in biopharmaceutical comparability studies. Journal of pharmaceutical sciences, 100(6), 2071-2086.

Hourdel, V. et al. (2016). Memhdx: an interactive tool to expedite the statistical validation and visualization of large hdx-ms datasets. Bioinformatics, 32(22), 3413-3419.

Jia, R. et al. (2020). Hydrogen-deuterium exchange mass spectrometry captures distinct dynamics upon substrate and inhibitor binding to a transporter. Nature communications, 11(1), 1-10.

Kammari, R. et al. (2020). Effects of secondary structure on solid-state hydrogen-deuterium exchange in model alpha-helix and beta-sheet peptides. Molecular Pharmaceutics, 17(9), $3501-3512$.

Katta, V. et al. (1993). Hydrogen/deuterium exchange electrospray ionization mass spectrometry: a method for probing protein conformational changes in solution. Journal of the American Chemical Society, 115(14), 6317-6321.

Kirisako, T. et al. (2006). A ubiquitin ligase complex assembles linear polyubiquitin chains. The EMBO journal, 25(20), 4877-4887.

Konermann, L. et al. (2011). Hydrogen exchange mass spectrometry for studying protein structure and dynamics. Chemical Society Reviews, 40(3), 1224-1234.

Lau, A. M. et al. (2020). Deuteros 2.0: peptide-level significance testing of data from hydrogen deuterium exchange mass spectrometry. Bioinformatics.

Liu, S. et al. (2011). Hdx-analyzer: a novel package for statistical analysis of protein structure dynamics. BMC bioinformatics, 12(1), 1-10.

Love, M. I. et al. (2014). Moderated estimation of fold change and dispersion for rna-seq data with deseq2. Genome biology, 15(12), 1-21.

Marquardt, D. W. (1963). An algorithm for least-squares estimation of nonlinear parameters. Journal of the society for Industrial and Applied Mathematics, 11(2), 431-441.

Masson, G. R. et al. (2017). An overview of hydrogen deuterium exchange mass spectrometry (hdx-ms) in drug discovery. Expert opinion on drug discovery, 12(10), 981-994.

Orengo, C. A. et al. (1999). From protein structure to function. Current opinion in structural biology, $\mathbf{9}(3), 374-382$.

Pascal, B. D. et al. (2012). Hdx workbench: software for the analysis of $\mathrm{h} / \mathrm{d}$ exchange ms data. Journal of the American Society for Mass Spectrometry, 23(9), 1512-1521. 
Ramsay, J. O. et al. (2007). Applied functional data analysis: methods and case studies. Springer.

Rey, M. et al. (2014). Mass spec studio for integrative structural biology. Structure, 22(10), $1538-1548$.

Ritchie, M. E. et al. (2015). limma powers differential expression analyses for rna-sequencing and microarray studies. Nucleic acids research, 43(7), e47-e47.

Sauve, V. et al. (2018). Mechanism of parkin activation by phosphorylation. Nature structural $\&$ molecular biology, 25(7), 623-630.

Schall, R. (1991). Estimation in generalized linear models with random effects. Biometrika, 78(4), 719-727.

Slysz, G. W. et al. (2009). Hydra: software for tailored processing of $\mathrm{h} / \mathrm{d}$ exchange data from ms or tandem ms analyses. Bmc Bioinformatics, 10(1), 1-14.

Smyth, G. K. (2004). Linear models and empirical bayes methods forassessing differential expression in microarray experiments. Statistical Applications in Genetics and Molecular Biology, 3(1).

Spratt, D. E. et al. (2014). Rbr e3 ubiquitin ligases: new structures, new insights, new questions. Biochemical journal, 458(3), 421-437.

Tsai, Y.-C. I. et al. (2020). Single-domain antibodies as crystallization chaperones to enable structure-based inhibitor development for rbr e3 ubiquitin ligases. Cell chemical biology, 27(1), 83-93.

Zhang, N. et al. (2020). Hd-explosion: visualization of hydrogen-deuterium exchange data as chiclet and volcano plots with statistical filtering. Bioinformatics.

\section{Supplementary Material}

\subsection{HOIP epitope mapping continued}

Here, we plot the peptide that had significantly altered deuterium kinetics. For some dAbs no such cases were found. The dAbs are subscripted by their molar concentration with respect to HOIP-RBR. No significant results are reported for dAb3, dAb6, dAb14 
bioRxiv preprint doi: https://doi org/10.1101/2021.11.02 466892. this version posted November 4, 2021. The copyright holder for this preprint (which was not certified by peer review) is the author/funder, who has granted bioRxiv a license to display the preprint in perpetuity. It is made available under aCC-BY 4.0 International license.
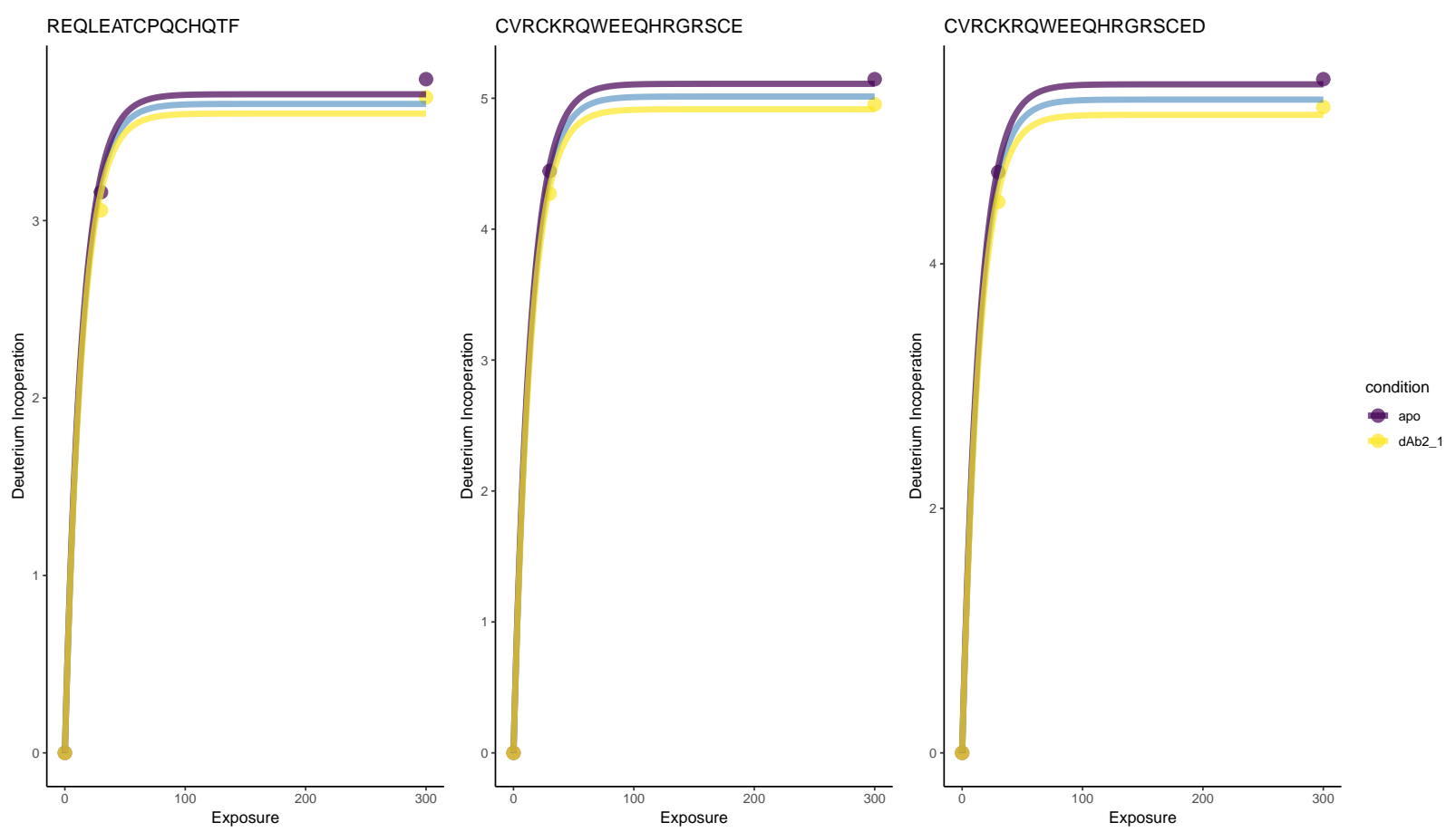

Figure $7: \mathrm{dAb} 2 \cdot \mathrm{b}=0.07, \mathrm{FDR}<0.05$

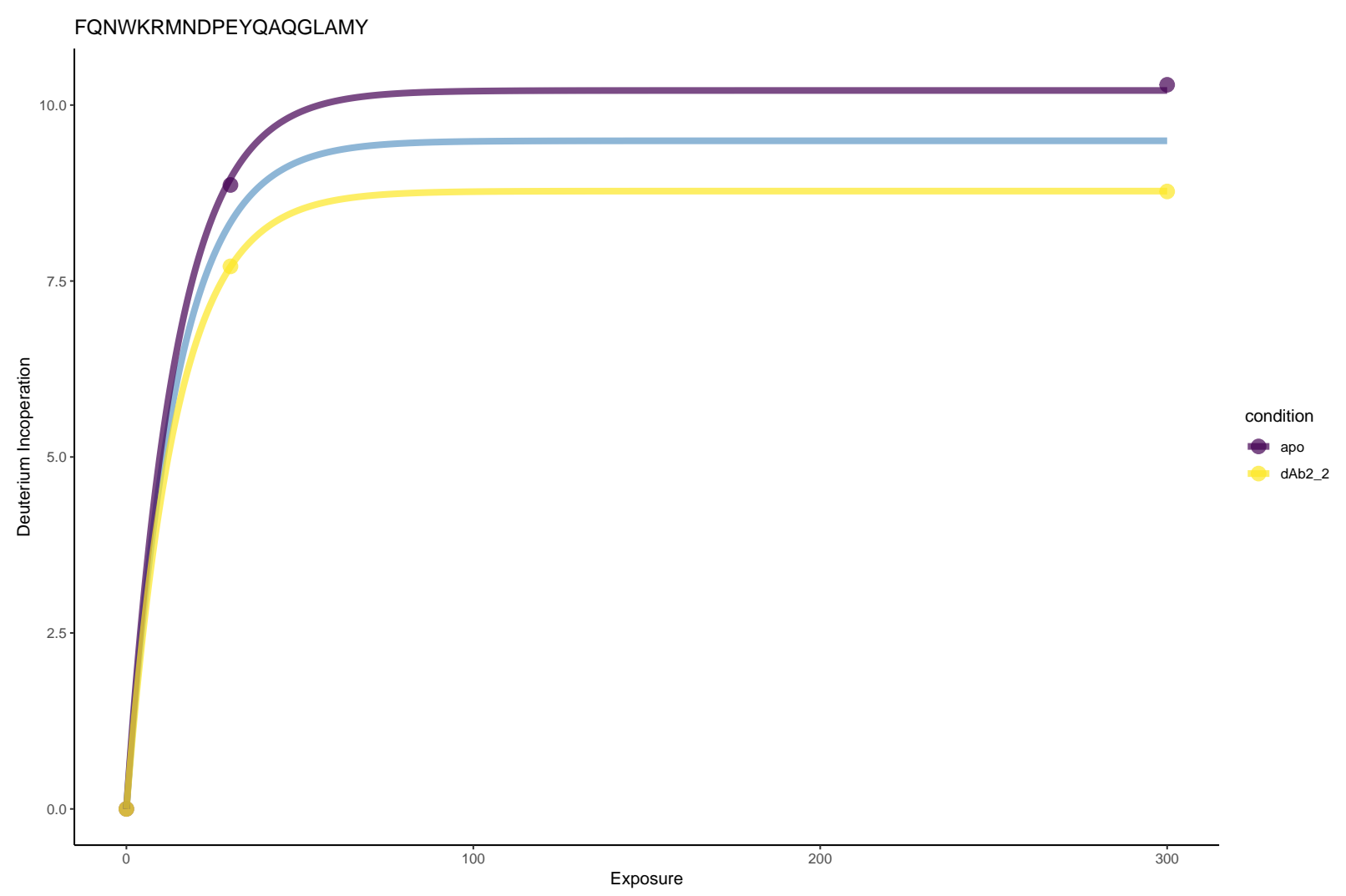

Figure 8: $\mathbf{d A b} 2_{2} \cdot \mathbf{b}=0.07, \mathrm{FDR}<0.05$ 
bioRxiv preprint doi: https://doi org/10.1101/2021.11.02 466892 this version posted November 4 2021. The copyright holder for this preprint (which was not certified by peer review) is the author/funder, who has granted bioRxiv a license to display the preprint in perpetuity. It is made available under aCC-BY 4.0 International license.
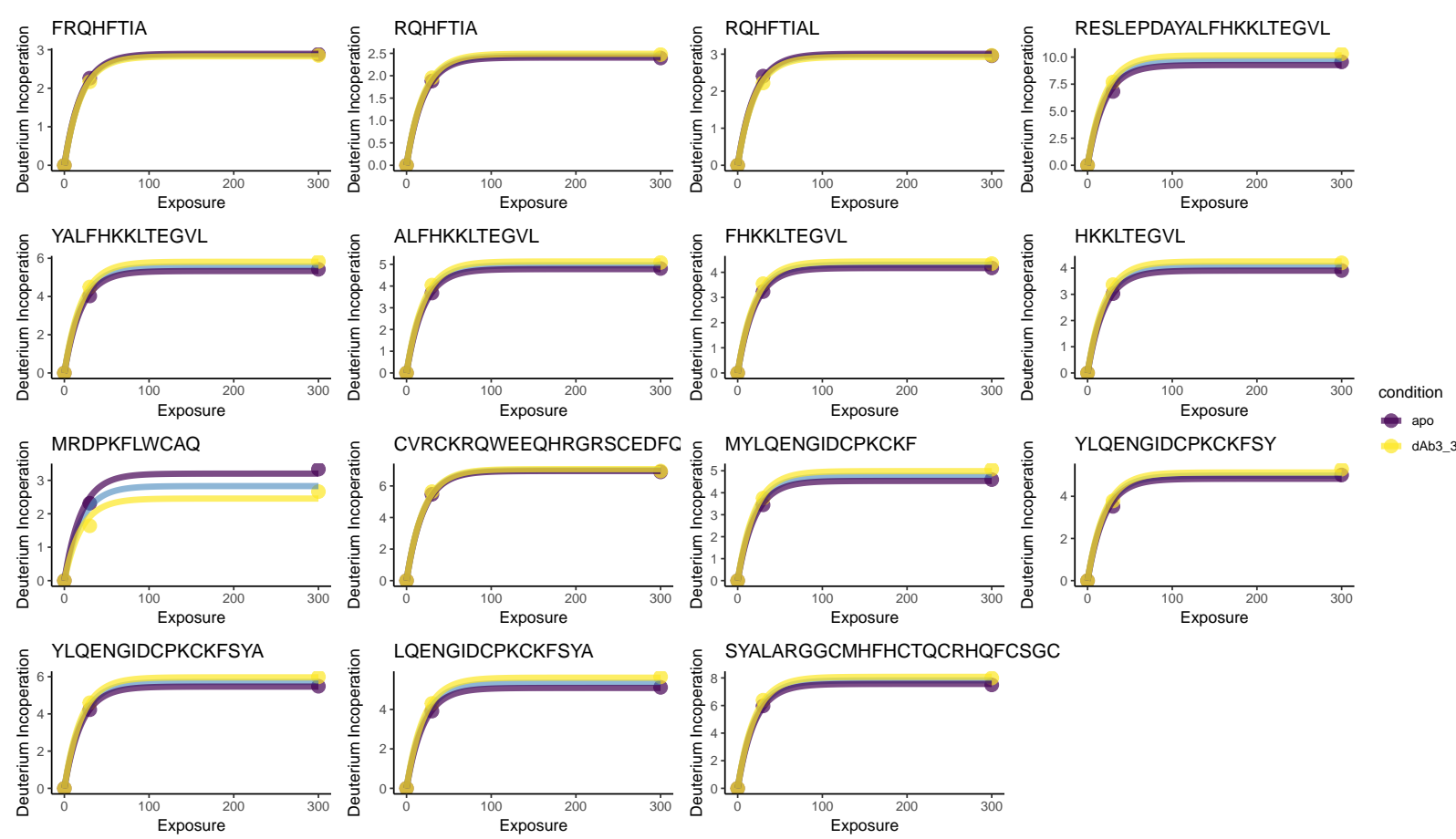

Figure 9: dAb18 $. \mathbf{b}=0.05, \mathrm{FDR}<0.05$
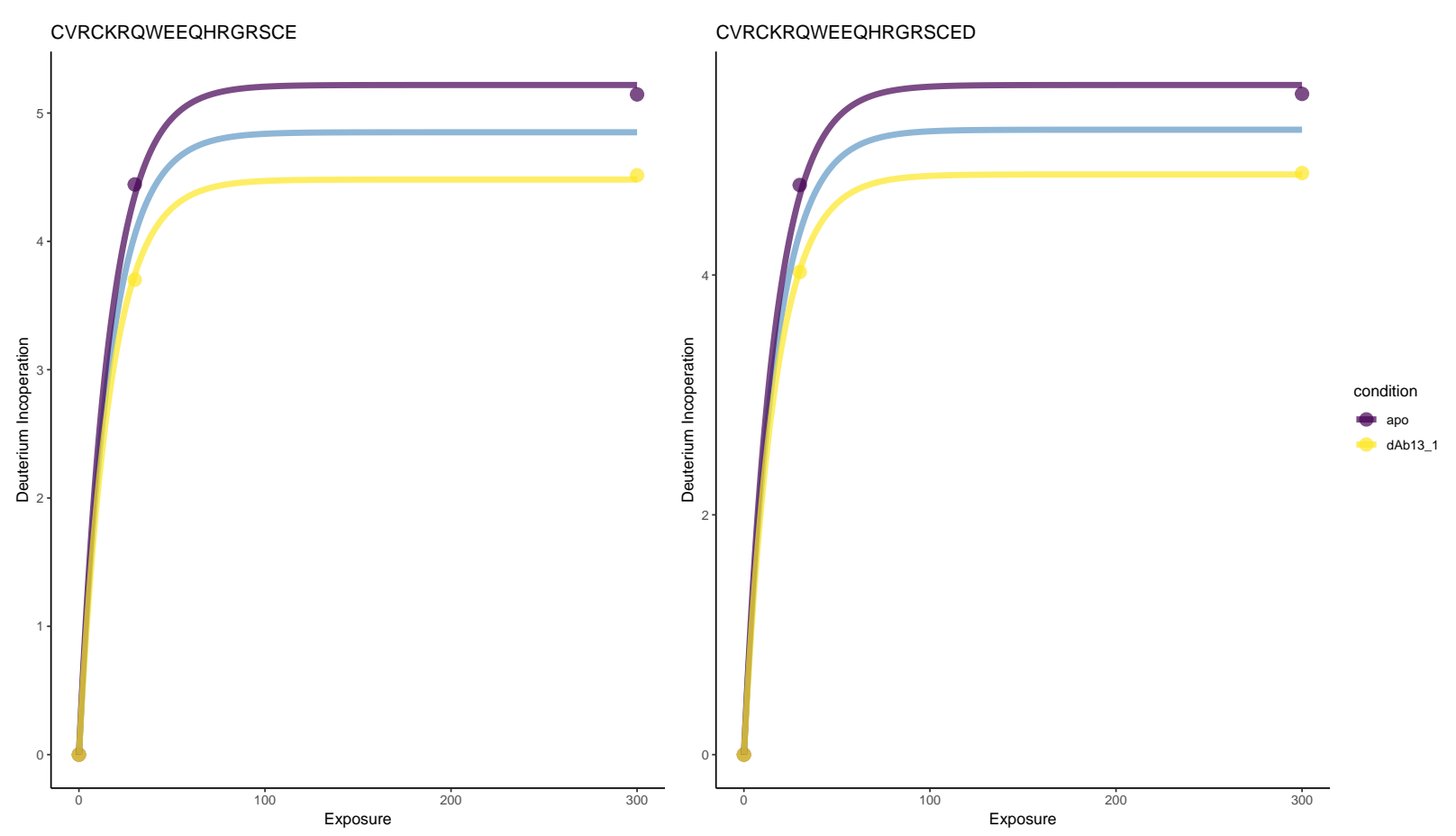

Figure 10: $\mathbf{d A b} 13_{1} \cdot \mathbf{b}=0.06, \mathrm{FDR}<0.05$ 
bioRxiv preprint doi: https://doi org/10.1101/2021.11.02.466892; this version posted November 4, 2021. The copyright holder for this preprint (which was not certified by peer review) is the author/funder, who has granted bioRxiv a license to display the preprint in perpetuity. It is made available under aCC-BY 4.0 International license.
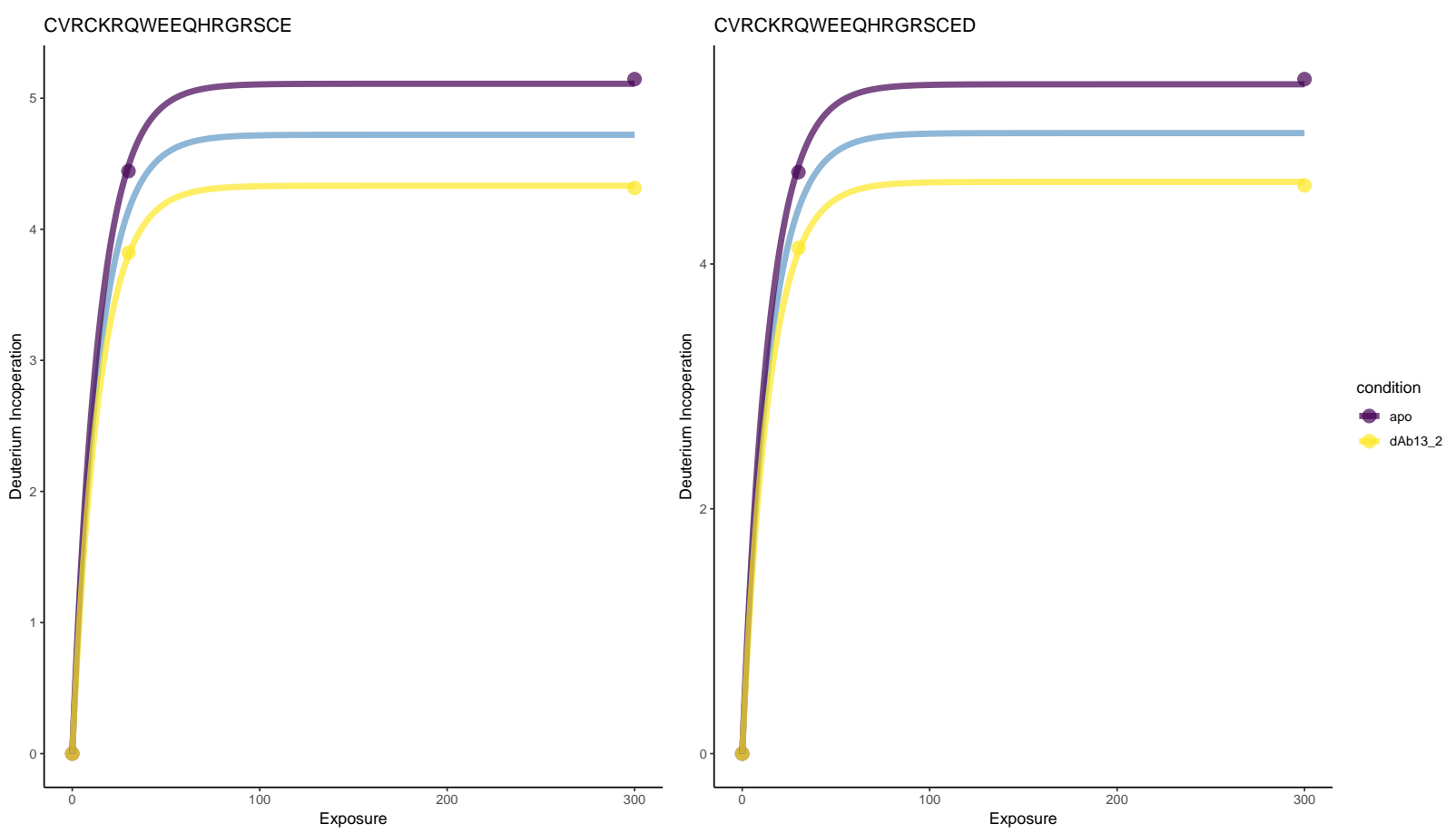

Figure 11: $\mathrm{dAb} 13_{2} \cdot \mathrm{b}=0.07, \mathrm{FDR}<0.05$ 
bioRxiv preprint doi: https://doi org/10.1101/2021.11.02 466892; this version posted November 4, 2021. The copyright holder for this preprint (which was not certified by peer review) is the author/funder, who has granted bioRxiv a license to display the preprint in perpetuity. It is made available under aCC-BY 4.0 International license.
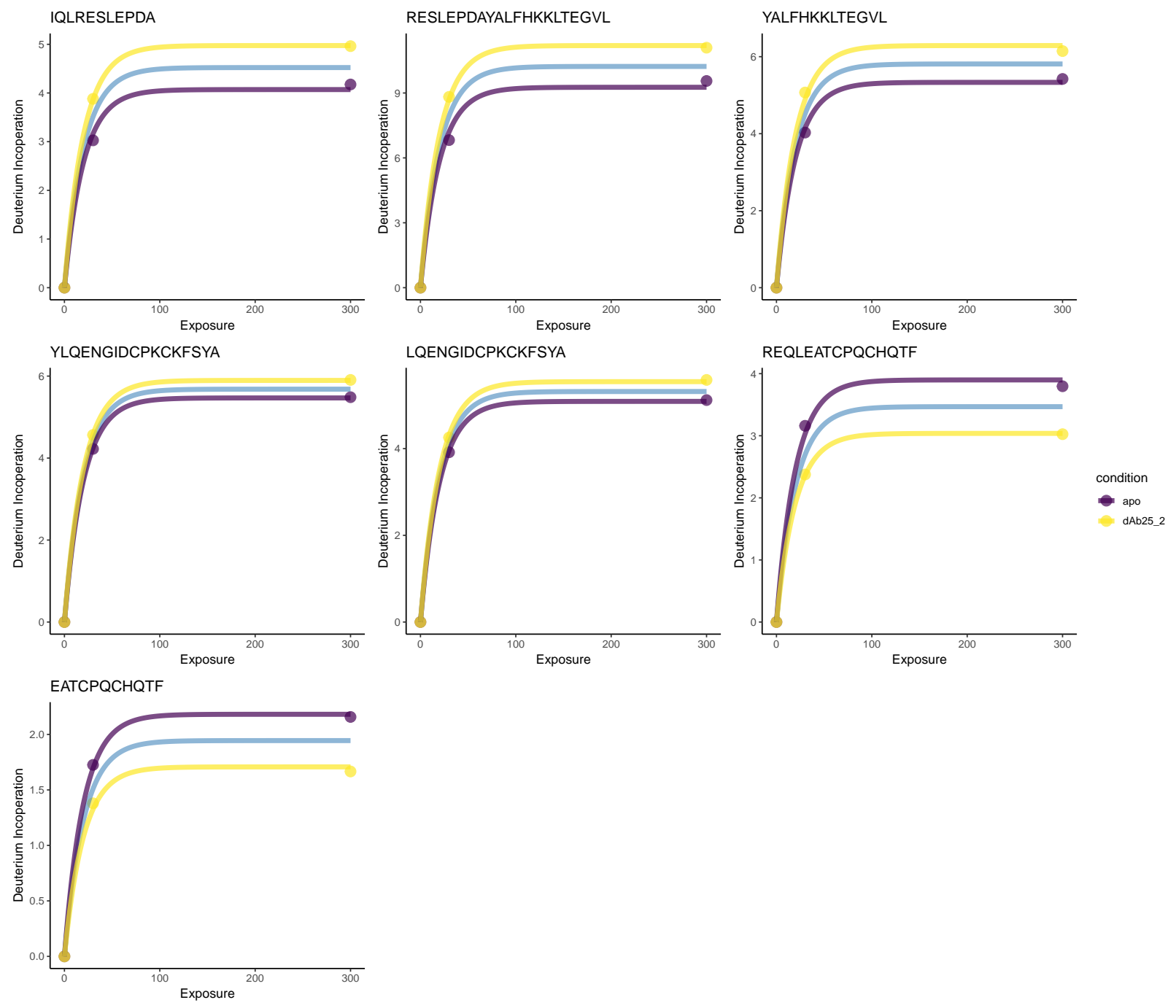

Figure 12: $\mathbf{d A b} 25_{2} \cdot \mathbf{b}=0.05, \mathrm{FDR}<0.05$ 
bioRxiv preprint doi: https://doi org/10.1101/2021.11.02 466892. this version posted November 4, 2021. The copyright holder for this preprint (which was not certified by peer review) is the author/funder, who has granted bioRxiv a license to display the preprint in perpetuity. It is made available under aCC-BY 4.0 International license.
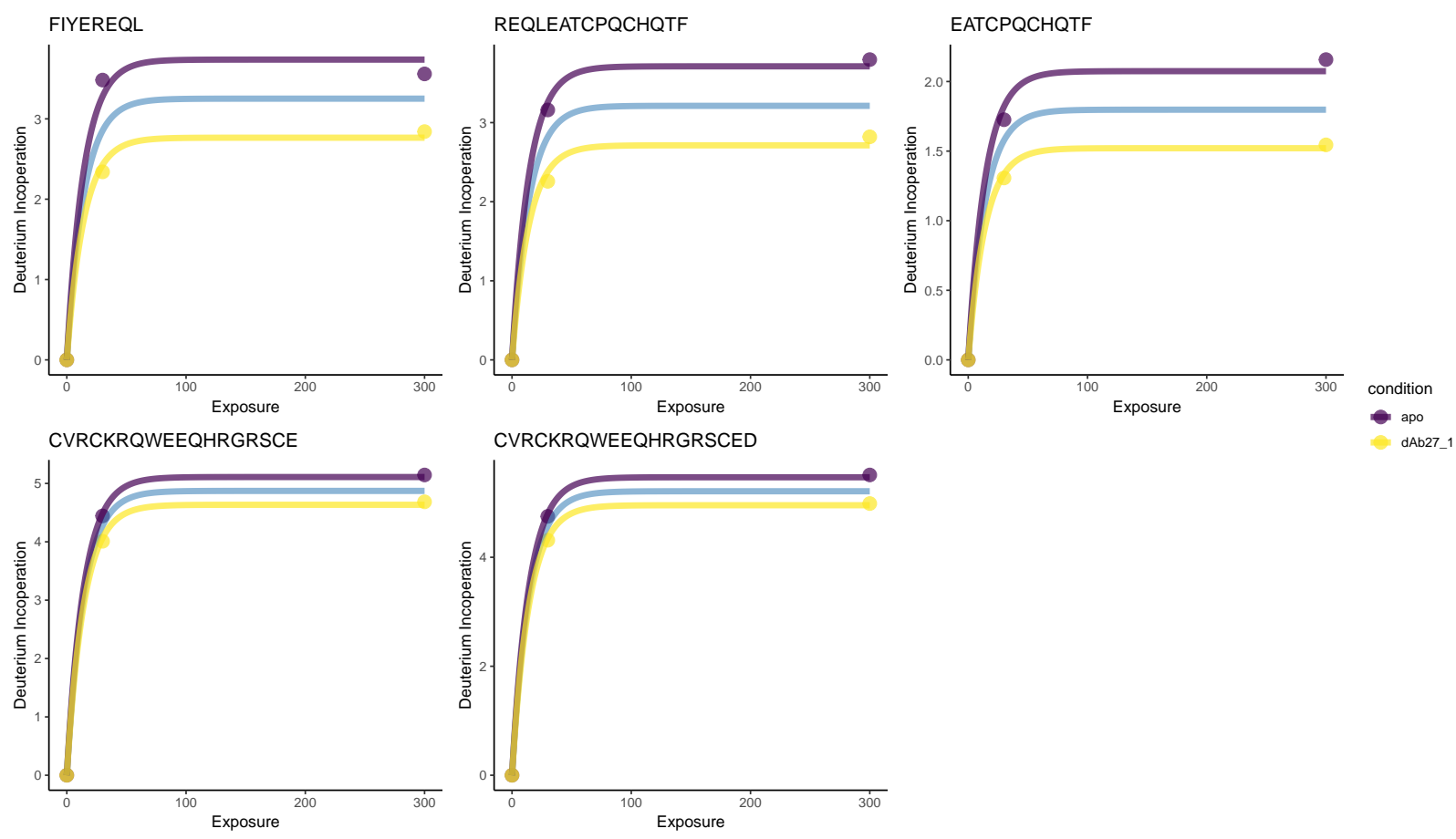

Figure 13: $\mathbf{d A b} 27_{1} \cdot \mathbf{b}=0.07, \mathrm{FDR}<0.06$
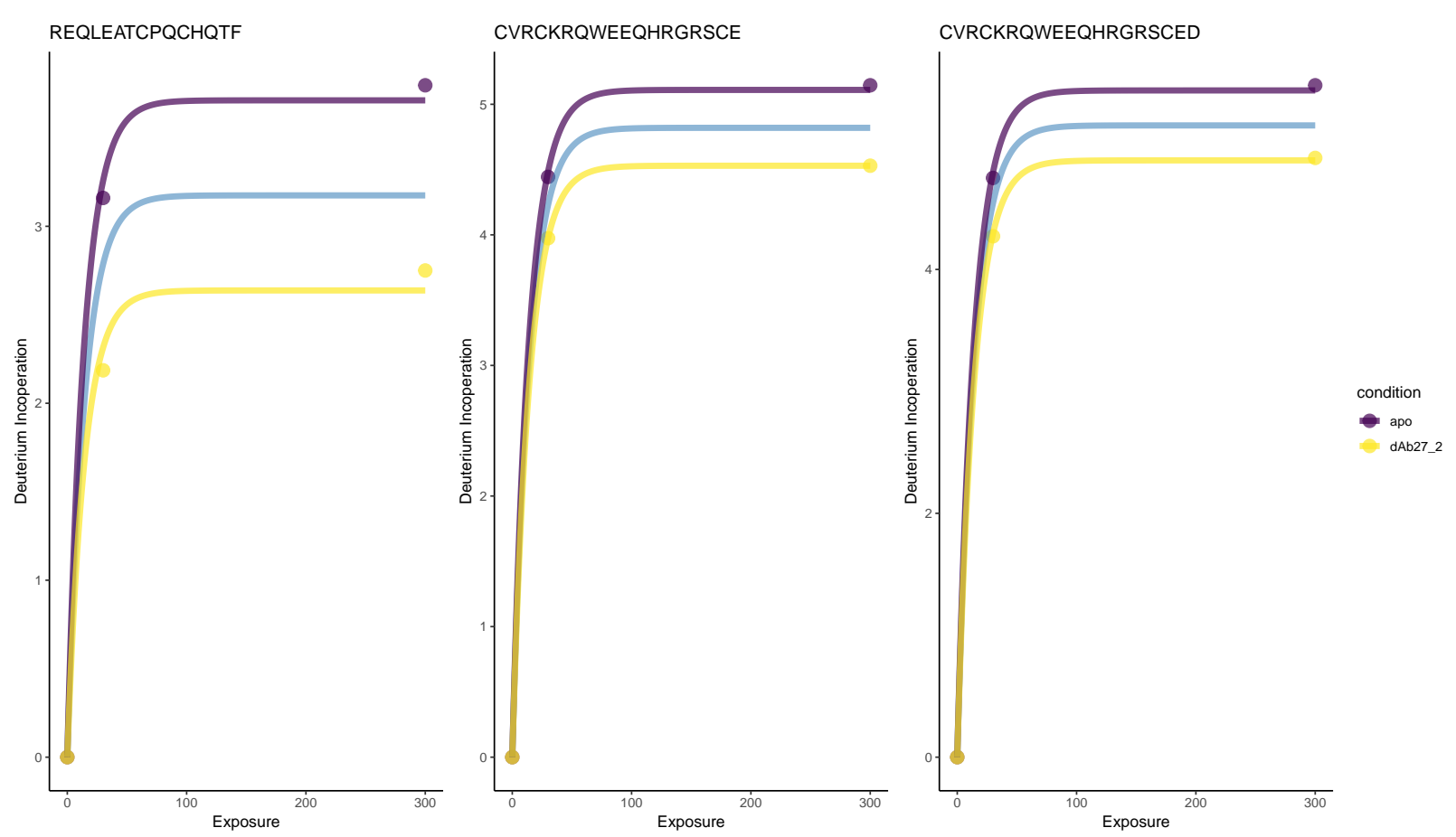

Figure 14: $\mathbf{d A b} 27_{2} \cdot \mathbf{b}=0.07, \mathrm{FDR}<0.05$ 
bioRxiv preprint doi: https://doi. org/10.1101/2021.11.02 466892. this version posted November 4, 2021. The copyright holder for this preprint (which was not certified by peer review) is the author/funder, who has granted bioRxiv a license to display the preprint in perpetuity. It is made available under aCC-BY 4.0 International license.
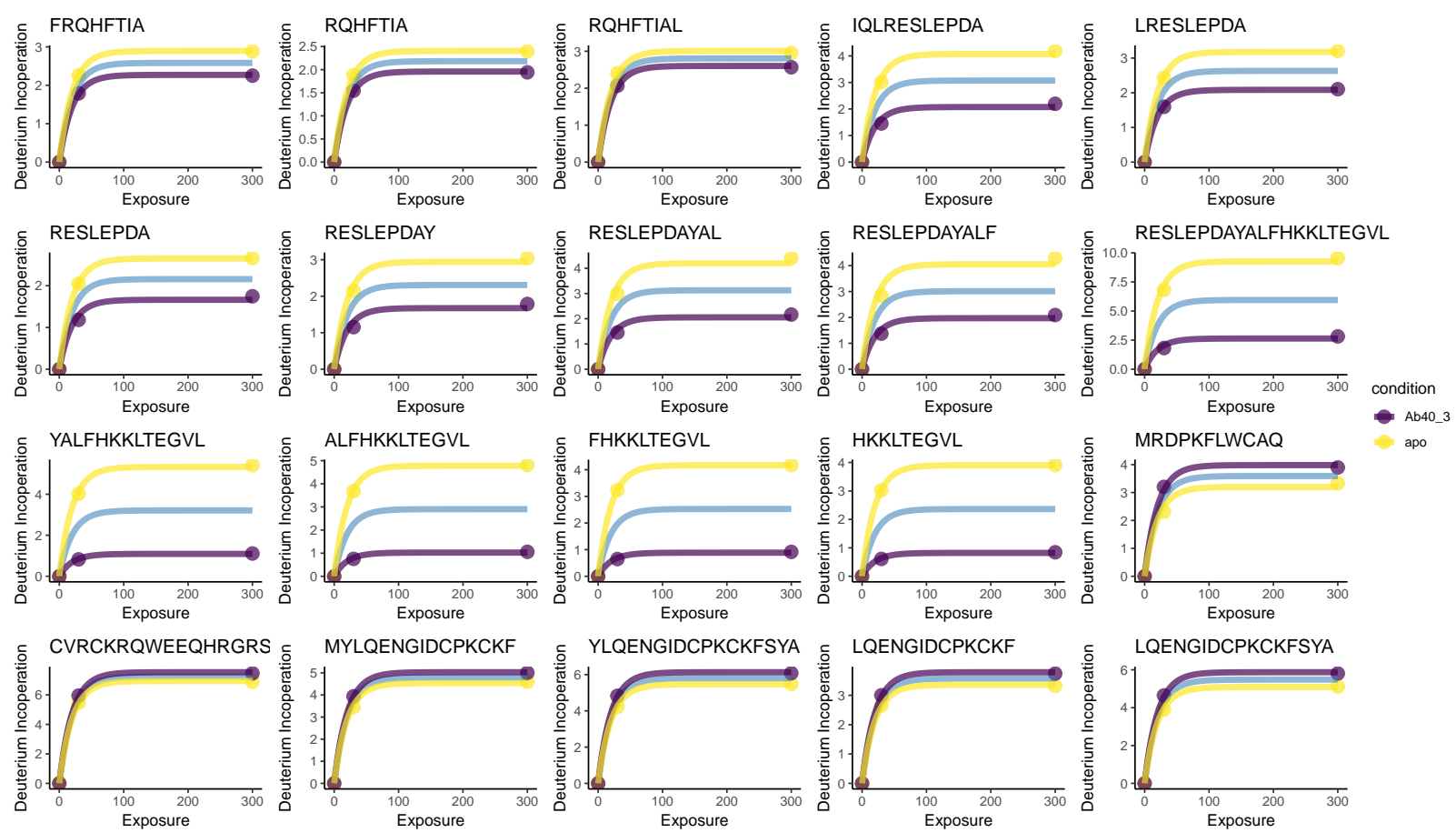

Figure 15: $\mathbf{d A b} 40_{3} \cdot \mathrm{b}=0.05, \mathrm{FDR}<0.05$

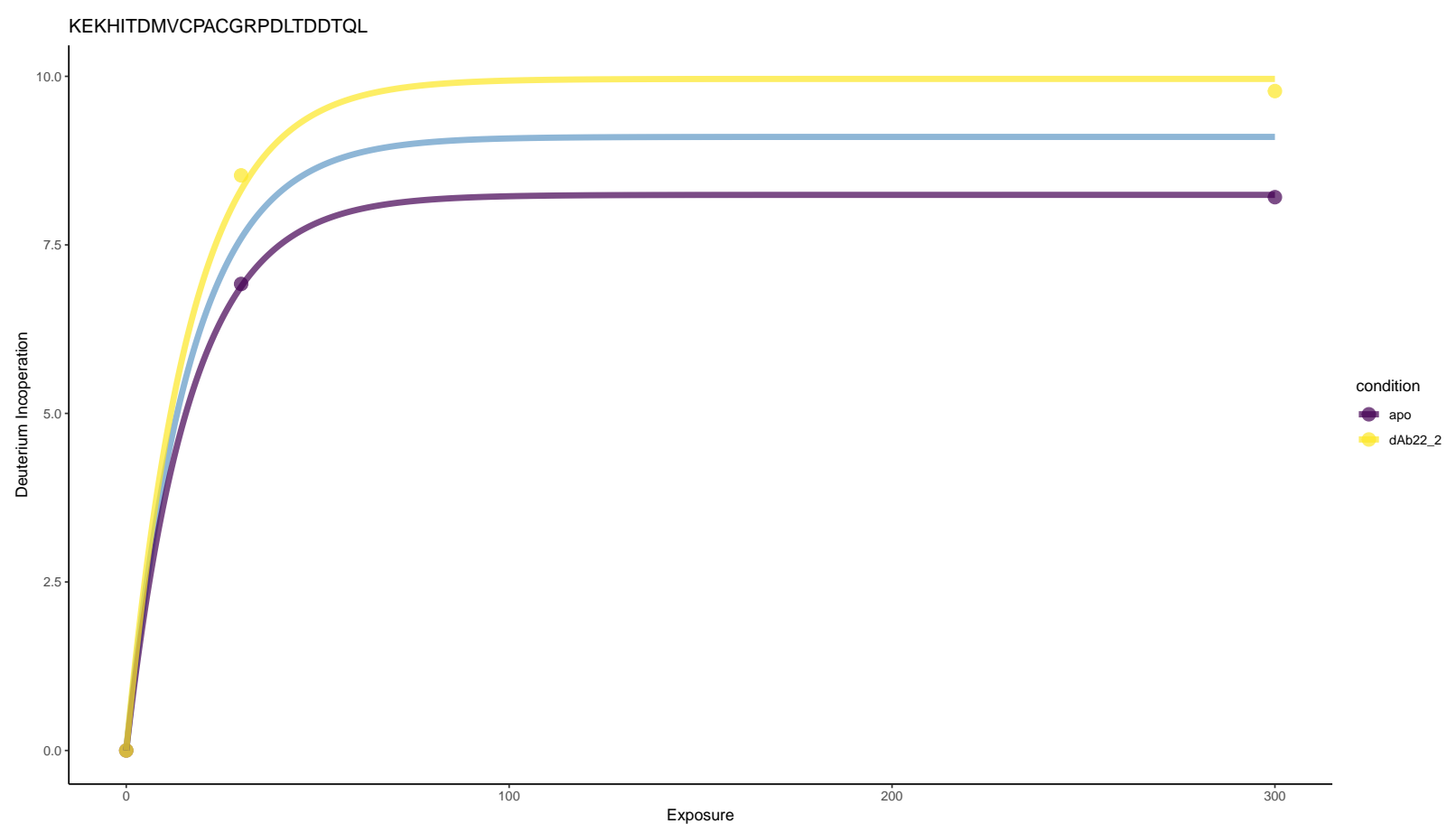

Figure 16: $\mathbf{d A b} 22_{2} \cdot \mathrm{b}=0.06, \mathrm{FDR}<0.05$ 
bioRxiv preprint doi: https://doi org/10.1101/2021.11.02 466892. this version posted November 4, 2021. The copyright holder for this preprint (which was not certified by peer review) is the author/funder, who has granted bioRxiv a license to display the preprint in perpetuity. It is made available under aCC-BY 4.0 International license.
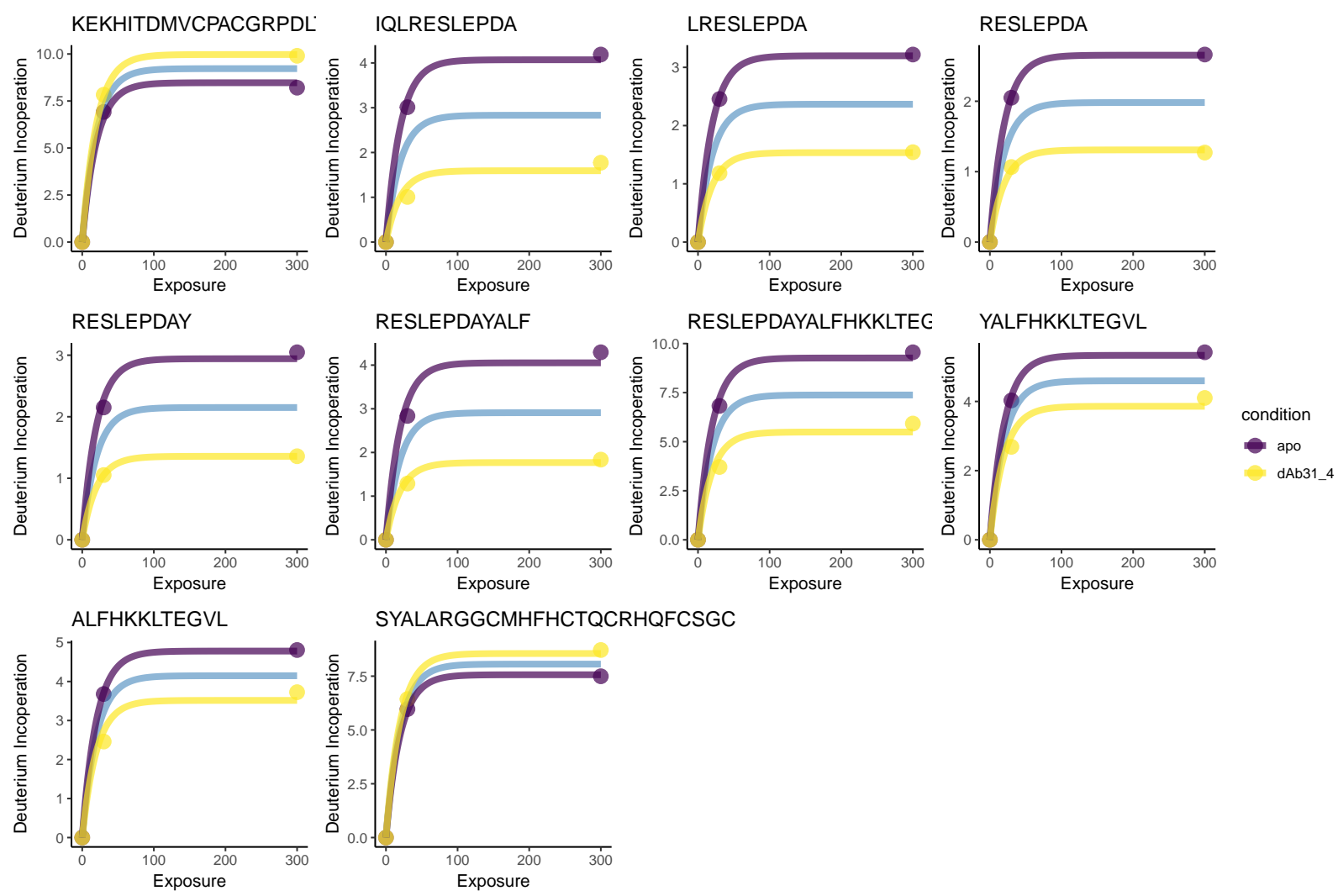

SYALARGGCMHFHCTQCRHQFCSGC

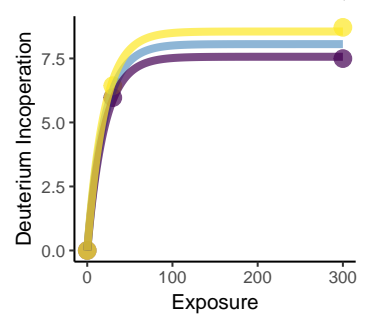

Figure 17: $\mathbf{d A b} 31_{4} \cdot \mathrm{b}=0.05, \mathrm{FDR}<0.05$ 
bioRxiv preprint doi: https://doi org/10.1101/2021.11.02 466892. this version posted November 4, 2021 . The copyright holder for this preprint (which was not certified by peer review) is the author/funder, who has granted bioRxiv a license to display the preprint in perpetuity. It is made available under aCC-BY 4.0 International license.
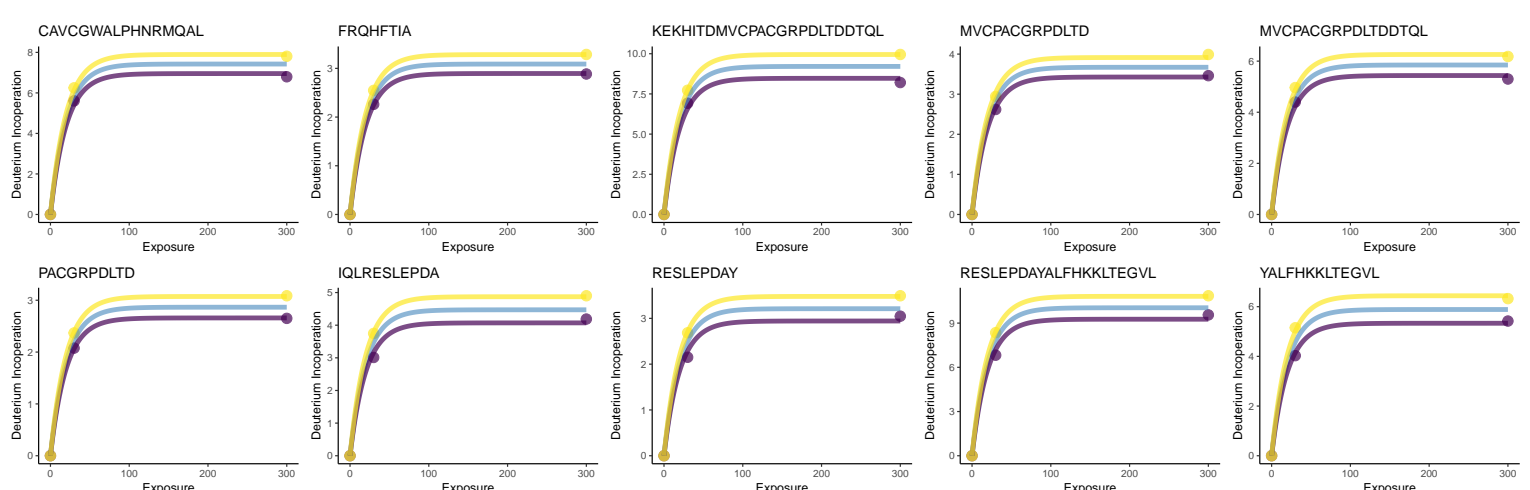

YALFHKKLTEGVL
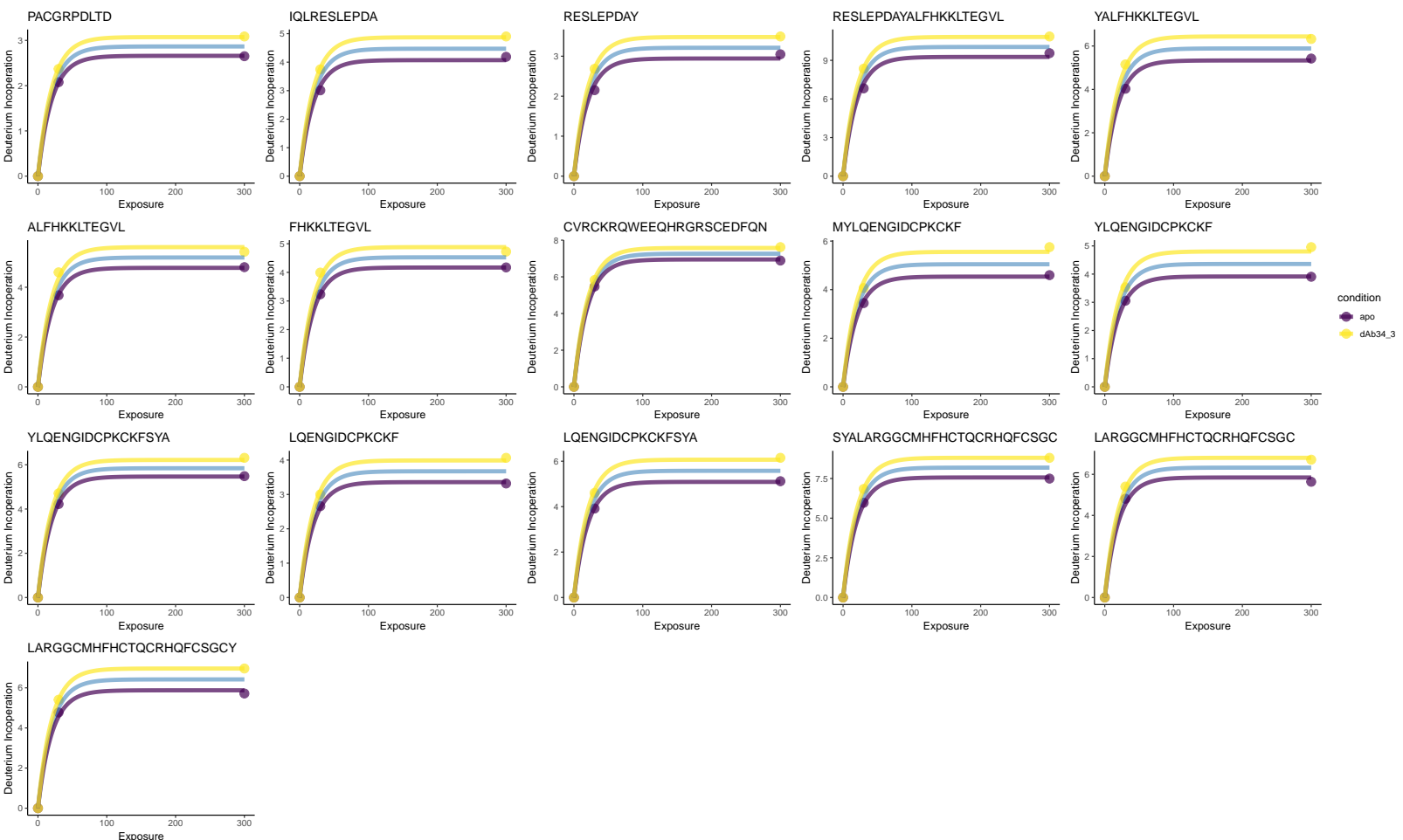

Figure 18: $\mathbf{d A b} 34_{3} . \mathrm{b}=0.05, \mathrm{FDR}<0.05$ 


\subsection{Further visualisation of HOIP-RBR-dAB25}

Alongside the Manhattan plot shown in the main text, we can plot significance on a standard peptide coverage plot. This allows us to obtain another global and spatial visualisation of the significantly altered peptides. This allows us to quickly identify support for our claims by examining whether overlapped peptides are also significantly perturbed, which can be tricky using a Manhattan plot. A number of factor can lead to an overlapping peptide not being declared significant and not declaring significance should not be considered evidence of the null.

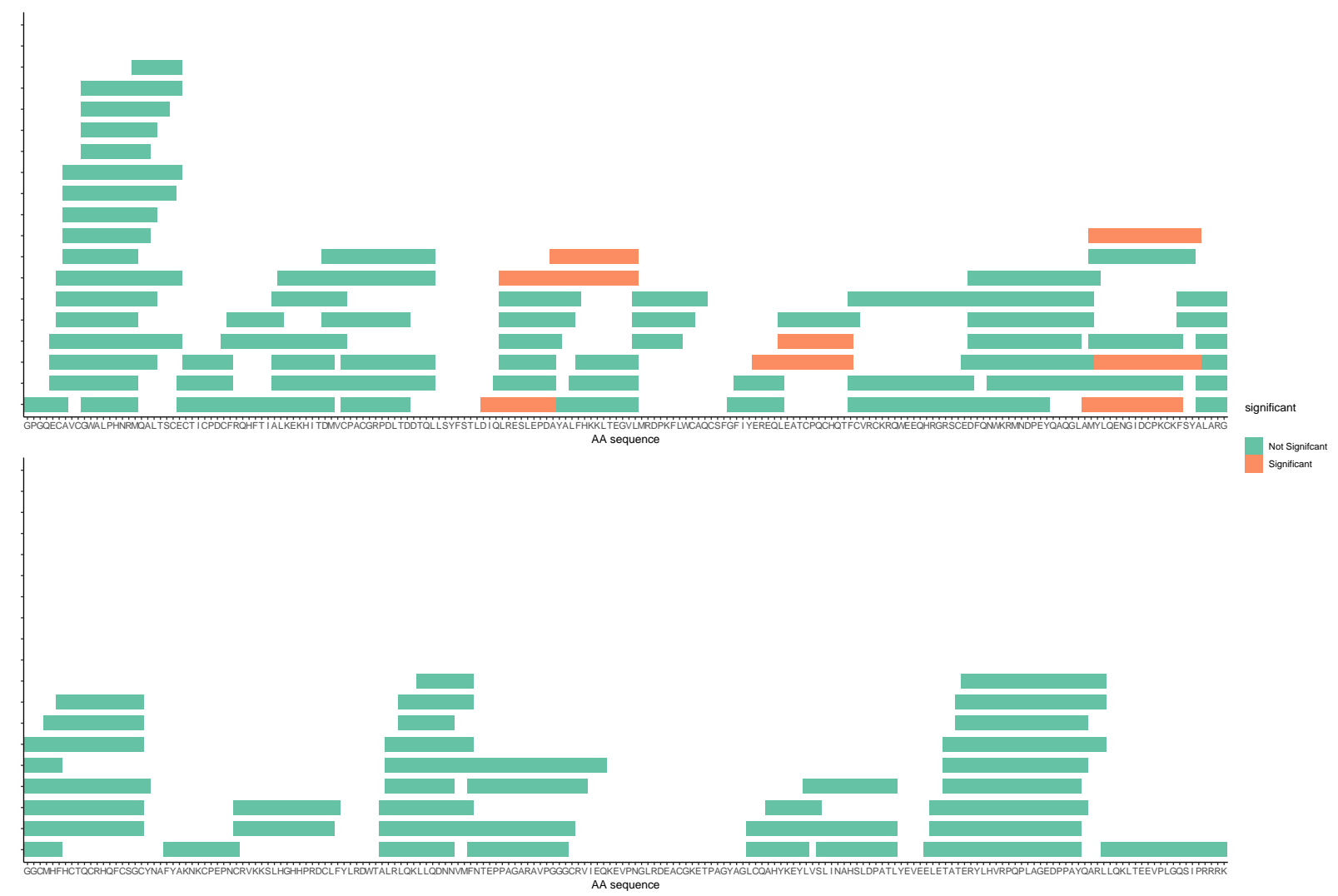

Figure 19: Peptide coverage plot with significance overlaid for dAb25.

\subsection{Global visualisation of multi-antibody Epitope mapping}

Alongside the functional uptake plots and peptide coverage plots, we can visualise residue level results by obtaining a score for each peptide. This is obtained using the harmonic mean of the $p$-values at each residue (see methods). We can then also plot all dAbs used in an experiment alongside each other. 
bioRxiv preprint doi: https://doi.org/10.1101/2021.11.02.466892; this version posted November 4, 2021. The copyright holder for this preprint (which was not certified by peer review) is the author/funder, who has granted bioRxiv a license to display the preprint in perpetuity. It is made available under aCC-BY 4.0 International license.

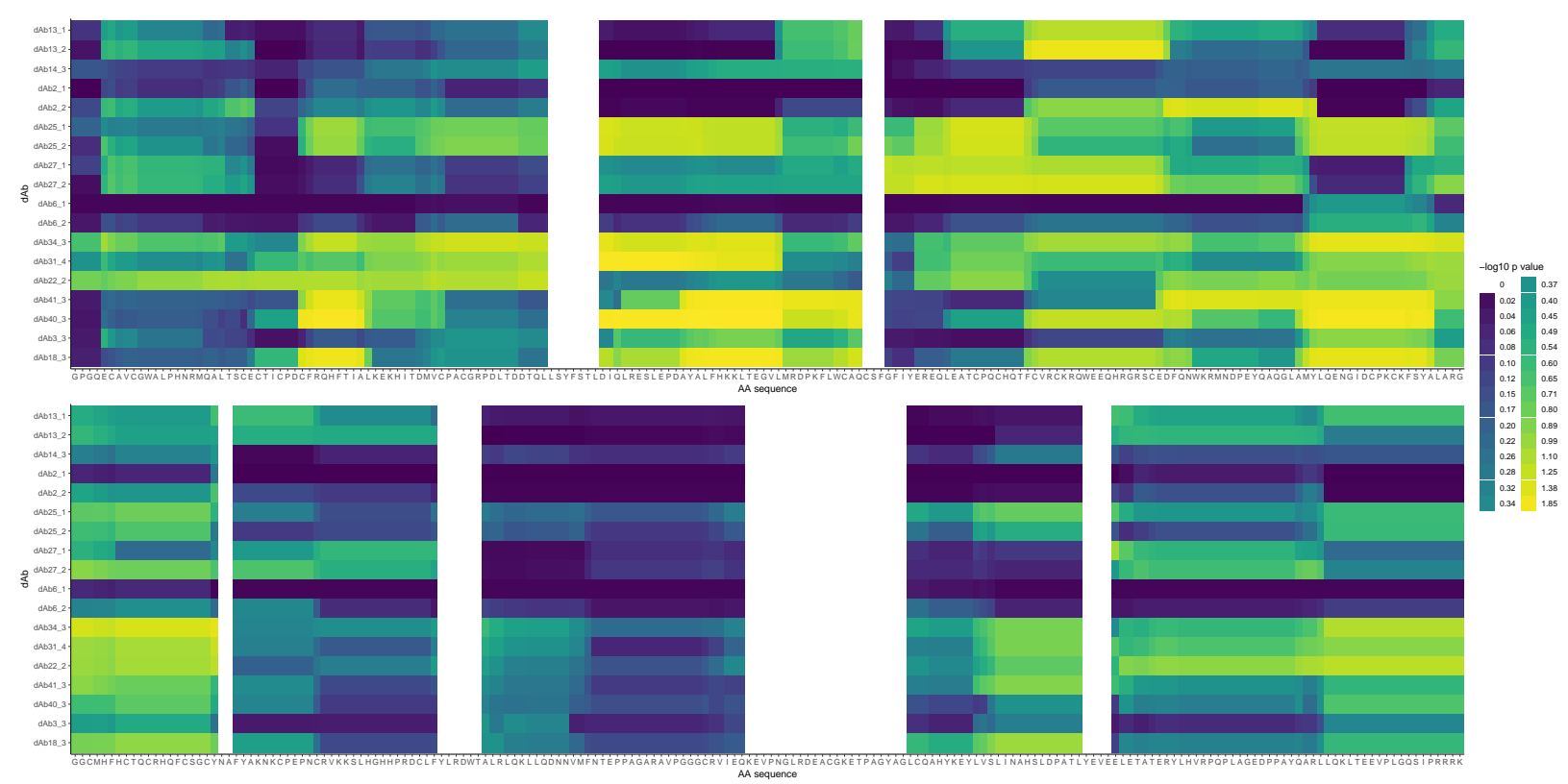

Figure 20: Heatmap showing residue level significance for all antibodies. Residue level visualisation plotted as a heatmap (see methods), for all dAbs used in the experiment. 